\title{
ON THE ASYMPTOTIC SOLUTIONS OF A CLASS OF ORDINARY DIFFERENTIAL EQUATIONS OF THE FOURTH ORDER, WITH SPECIAL REFERENCE TO AN EQUATION OF HYDRODYNAMICS $\left({ }^{1}\right)$
}

\author{
BY \\ RUDOLPH E. LANGER
}

1. Introduction. The differential equation with which this investigation is concerned is of the form

$$
\mathscr{L}(w)=0,
$$

with

$$
\mathcal{L}(w) \equiv w^{\mathrm{iv}}+\lambda^{2}\left\{P(z, \lambda) w^{\prime \prime}+Q(z, \lambda) w^{\prime}+R(z, \lambda) w\right\} .
$$

The differentiations (indicated by accents or by a Roman superscript) are with respect to the (complex) variable $z$. For this variable a domain will be specified. The symbol $\lambda$ in the equation signifies a parameter. This is assumed to be large in absolute value and is dealt with also as being complex. The functions $P(z, \lambda), Q(z, \lambda)$, and $R(z, \lambda)$, for such $z$ and $\lambda$ are assumed to be expressible as power series in $1 / \lambda$ with coefficients that are functions of $z$ thus

$$
\begin{aligned}
& P(z, \lambda)=\sum_{n=0}^{\infty} \frac{P_{n}(z)}{\lambda^{n}}, \\
& Q(z, \lambda)=\sum_{n=0}^{\infty} \frac{Q_{n}(z)}{\lambda^{n}}, \\
& R(z, \lambda)=\sum_{n=0}^{\infty} \frac{R_{n}(z)}{\lambda^{n}} .
\end{aligned}
$$

Equations (1.1) in which $\lambda$ is real, or pure imaginary, or in which the functions $P(z, \lambda), Q(z, \lambda)$ and $R(z, \lambda)$ are merely polynomials in $1 / \lambda$, or even just functions of $z$, are, of course, included as special cases.

The salient feature of the whole investigation is that the domain of the (complex) variable $z$ in which the differential equation (1.1) is considered, is one that includes a zero of the coefficient $P_{0}(z)$. Such a zero is called a turning point (or a transition point) of the differential equation. The discussion is restricted to the case in which this zero is simple.

In a $z$-region that includes a turning point, the solutions of such a differential equation as (1.1) in general depend upon a parameter, such as $\lambda$, in so

Presented to the Society, February 25, 1956; received by the editors February 10, 1956.

(1) This research was supported by the United States Air Force through the Office of Scientific Research, Air Research and Development Command. 
intricate a way, as to have quite distinct functional forms in different parts of the region. What these forms are is not easily determined, for the asymptotic methods that may be regarded as classical in the theory of ordinary linear differential equations fail completely in such regions. Yet it is precisely there that many differential equations which arise in physical applications are of the greatest interest and importance. This is familiarly the case with certain differential equations of the second order that are central to the theories of micro-wave propagation and quantum mechanics. An instance from differential equations of the fourth order, namely from ones of the form (1.1), is the so-called "Orr-Sommerfeld" equation of hydrodynamics,

$$
\begin{aligned}
\frac{d^{4} \psi}{d y^{4}}-\{i \alpha R[\omega(y)-c]+ & \left.2 \alpha^{2}\right\} \frac{d^{2} \psi}{d y^{2}} \\
& +\left\{i \alpha R\left[\alpha^{2}(\omega-c)+\frac{d^{2} \omega}{d^{2} y}\right]+\alpha^{4}\right\} \psi=0 .
\end{aligned}
$$

This is basic to the analysis of the stability of a laminar fluid flow. A characterization of the onset of turbulence in such a flow requires the forms of the solutions of this differential equation in a neighborhood of the point in which the function $[\omega(y)-c]$ vanishes, namely of the turning point. The existing literature of the subject of the present paper has essentially all been motivated, more or less directly, by the need for the solution forms of the differential equation (1.4). I have in mind especially some papers by W. Wasow $[1 ; 2 ; 3]$, and by D. Meksyn $[4 ; 5]$. More extensive lists of references to hydrodynamical treatises and research papers are given by these authors.

Every linear differential equation of the form

$$
\sum_{n=0}^{k} \lambda^{n} a_{n}(z, \lambda) \frac{d^{k-n} w}{d z^{k-n}}=0
$$

in which the coefficients $a_{n}(z, \lambda)$ are analytic in $\lambda$ at $\lambda=\infty$, has associated with it an auxiliary algebraic equation, namely the equation

$$
\sum_{n=0}^{k} a_{n}(z, \infty) \chi^{k-n}=0 .
$$

The configuration of the roots of this auxiliary equation, and especially their multiplicities, have much to do with the asymptotic forms of the solutions of the differential equation. Except in the elementary case of constant coefficients, these roots, and hence the pattern of such multiplicities as occur among them, depend upon $z$. It is because this pattern is peculiar at a turning point, that such a point has especial significance with respect to the differential equation's solution forms.

The classical methods of asymptotic solution for equations of the form (1.1) are applicable only in z-regions over which the auxiliary roots maintain 
an unchanging pattern, namely over which distinct roots remain distinct, and multiple roots, if any, maintain their multiplicities identically in $z$. A turning point, on the other hand, is, by definition, one at which an isolated multiplicity occurs. The configuration of the roots is therefore different at such a point than it is elsewhere in the neighborhood. In the instance of the differential equation (1.1) the auxiliary equation is

$$
\chi^{4}+P_{0}(z) \chi^{2}=0 .
$$

In a deleted neighborhood of the turning point this has two roots that are simple, and another that is identically double. At the turning point, the roots all coincide in a single four-fold multiplicity.

The limiting form of the differential equation (1.1) as $\lambda \rightarrow \infty$ is the differential equation of the second order

$$
P_{0}(z) w^{\prime \prime}+Q_{0}(z) w^{\prime}+R_{0}(z) w=0 .
$$

For the differential equation (1.1) the turning point is an ordinary point. For the equation (1.7), on the other hand, it may well be a regular singular point, and is in fact so unless the functions $Q_{0}(z)$ and $R_{0}(z)$ as well as $P_{0}(z)$ vanish there. Of the two exponents of the equation (1.7) relative to this point, one is 0 . The other one we shall designate by $\rho$. The necessity of distinguishing the cases in which $\rho$ is an integer from those in which it is not, is familiar in connection with the differential equation (1.7). We shall see that it carries over into the discussion of the equation (1.1). Indeed this circumstance has largely dictated the organization of the present paper. A differential equation (1.1) for which $\rho$ is not an integer is designated in the following as being regular, and the theory which is presented for such equations is called the general theory. A differential equation for which $\rho$ is an integer is termed irregular. Such equations we have classified into four categories, on the basis of the adaptations of method that needed to be made for them. For equations of three of these categories the solution forms are obtained. Equations of the remaining category unfortunately appear to elude our method, at least in its present form.

The results of the paper may be summarized thus: Any $z$ in the domain specified for this variable, and any $\lambda$ of large modulus, lie within so-called associated regions. By this association an integer $h$ is identified, and for the $z$ and $\lambda$ of these regions four linearly independent solutions $w_{h, j}(z), j=0,1,2,3$, are singled out. The forms of these solutions are set forth by the Theorems $1,2,3$, and 4 , of $\S \S 13,14$, and 15 . Each of these forms consists of a certain exponential factor, and, aside from that, of an explicit part which is a polynomial in $1 / \lambda$ plus a remainder term which is of higher degree in $1 / \lambda$. The degree of this polynomial part, which in fact signifies the degree of explicitness of the solution forms obtained, can be arbitrarily pre-chosen. If this degree is chosen to be zero the dominant terms of the solution forms alone are ob- 
tained. An exponent $\delta$ which appears in the statements of the theorems has the value 0 for differential equations (1.1) that are regular or of the irregular categories defined in $\$ 17$. Its value for an equation of the category dealt with in $\$ 18$ is given in (18.1).

The hydrodynamical equation (1.4) is always irregular, as is any equation (1.1) in which $Q_{0}(z)$ vanishes at the turning point, and hence, of course, as is any equation (1.1) in which the term in the first derivative is lacking. The category of the equation depends upon its parameters and its coefficient function $\omega(y)$. The character of the computations by which the equation's category may be determined is indicated in $\$ \$ 19$ and 20 .

In the matter of method the present paper differs wholly from all others known to me on this subject. These latter all depend, in the first instance, upon applications of the method of steepest descent to the solutions of the given differential equation as these are expressed by Laplace contour integrals. They therefore depend upon the representability of the solutions in such a form, and are thus of very limited generality. They commonly require that $\arg \lambda$ be constant, that $P_{0}(z)$ be some special function, that $Q(z, \lambda)$ be vanishing and $R_{0}(z)$ nonvanishing, that $P(z, \lambda)$ and $R(z, \lambda)$ be linear polynomials in $1 / \lambda^{2}$ etc. The results obtained by them are explicit only to the extent of the dominant terms. No such specialized requirements figure in the present paper, and, as has already been said, the solution forms are obtained to an arbitrarily pre-assigned degree of explicitness.

The method of the present paper is an extension of one that I have previously developed in connection with differential equations of the second and third orders $[6 ; 7 ; 8 ; 9 ; 10]$. This depends upon the construction of a so-called related differential equation, which is one whose solution forms are known, and which, at the same time, resembles the given differential equation to an arbitrarily prescribed degree. From the known solution forms for the related equation, those for the given differential equation can be inferred. The construction of the related equation proceeds by certain formal algorithms. This is carried out in Part I of the paper. The inference of the solution forms is then a matter of rigorous analysis. This is carried out for the equations of regular type in Part II. Differential equations of irregular type are dealt with in Part III. The categories are there defined, and, to the extent that was feasible, the adaptations and modifications of theory by which the results of Part II are made applicable to these equations are there set forth.

The adjustments and hypotheses under which the discussion is to proceed are conveniently set down at this point. There is no loss of generality in locating the origin of $z$ at the turning point. For the convenience of it we shall do so. The hypotheses are then the following:

(i) The domain of the variable $z$ is a bounded closed region $R_{z}$ of the complex plane that includes the origin in its interior.

(ii) The functions $P_{0}(z)$ and $\int_{0}^{z} P_{0}^{1 / 2}(z) d z$ are nonvanishing in $R_{z}$ except at 
the origin. At the origin $P_{0}(z)$ has a zero that is simple, i.e.

$$
P_{0}(0)=0, \quad P_{0}^{\prime}(0) \neq 0 .
$$

(iii) The parameter $\lambda$ is bounded from zero, and is otherwise eligible to take on any complex values.

(iv) The coefficients $P_{n}(z), Q_{n}(z)$, and $R_{n}(z)$ of the series (1.3) are analytic in $R_{z}$ and these series, if they are infinite, are convergent when $|\lambda|$ is sufficiently large.

(v) The exponent of $\rho$ of the differential equation (1.7), relative to the origin, namely

$$
\rho=1-Q_{0}(0) / P_{0}^{\prime}(0)
$$

is not a negative integer.

Beyond those indicated by these explicit statements, there are certain limitations upon the region $R_{\mathbf{z}}$ both as to size and shape, that cannot be concisely summarized and set forth here. They will appear in the course of the discussion. Their significance is minor. The essential matter is that $R_{z}$ be a full neighborhood of the turning point, and this remains assured.

It should be noted that the stipulation by hypothesis (v) that $\rho$ be not a negative integer is not as restrictive as it may seem to be at first glance. For when $\rho$ is a negative integer, we may transfer the attention to the adjoint of the equation (1.1). This is a differential equation which is also of the form (1.1), but in which $\rho$ is a positive integer. The solution forms of the adjoint equation may thus be determinable, and from these, as is familiar, the solution forms of the given equation are readily obtainable.

\section{PART I. Formal AlgorithmS}

2. A method of undetermined coefficients. The processes for formally solving ordinary linear differential equations, for large values of a parameter $\lambda$, generally yield expressions in which there appear power series in $1 / \lambda$. When such expressions are obtainable, they cannot ordinarily be expected to be actual solutions of the differential equation, but only formal ones, due to the fact that the series they contain are divergent. It is, however, also widely true that truncations of these series, say at the terms in $1 / \lambda^{m}$, yield functions that represent actual solutions asymptotically, at least in suitably restricted domains of $\lambda$ and $\boldsymbol{x}$. The explicitness of these representations increases, of course, with the integer $m$. When $m=0$, namely when only the first terms of the power series are retained, the representations extend only to the so-called dominant terms.

We shall see that these remarks apply in particular to differential equations of the type (1.1). To say that we shall derive asymptotic representations that extend to an arbitrary degree of explicitness, is therefore to say that we may permit the positive integer $m$ to be arbitrarily chosen. That we shall do. 
And we shall assume from this point on that such a choice has been made once for all. The specifically chosen value of $m$ will be held to throughout the discussion.

The procedure upon which we will now enter may be outlined as follows: With $L(u)$ standing for a differential form

$$
L(u) \equiv u^{\prime \prime \prime}+\lambda^{2} p(z, \lambda) u^{\prime}+\lambda^{2} q(z, \lambda) u,
$$

which is of the third order, and in which the coefficients $p(z, \lambda)$ and $q(z, \lambda)$ are tentatively undetermined, the equation

$$
L^{\prime}(u)+\frac{r(z, \lambda)}{\lambda^{m+1}} L(u)=0,
$$

with any coefficient $r(z, \lambda)$ is a differential equation of the fourth order. Through the formula

$$
\eta=a(z, \lambda) u+\frac{1}{\lambda^{2}} \mathfrak{e}(z, \lambda) u^{\prime \prime},
$$

in which the coefficients $Q(z, \lambda)$ and $\mathfrak{e}(z, \lambda)$ are specific but momentarily undetermined there is associated with each solution $u_{j}$ of the equation (2.2) a function $\eta_{j}$. These functions $\eta_{j}$ are, in their turn, the solutions of a certain differential equation of the fourth order which we shall, in the end, designate as the related equation. We shall show that by a suitable term by term determination of the functions $p(z, \lambda), q(z, \lambda), a(z, \lambda)$, and $\mathfrak{e}(z, \lambda)$, as polynomials in $1 / \lambda$, we can make the coefficients of the related equation the same as those of the given differential equation to the extent of all terms of a lower power than the $(m+1)$ th in $1 / \lambda$.

The formalism by which this program is carried out constitutes Part I of this paper. It will be found, when this has been done, that the equation

$$
L(u)=0,
$$

is one for which the forms of three linearly independent solutions are obtainable from the literature. These solutions evidently also fulfill the equation (2.2). We shall then determine the function $r(z, \lambda)$ so as to make a fourth solution of the equation (2.2) known. The four solutions $u_{j}, j=0,1,2,3$ then yield through the formula (2.3) a corresponding set of four solutions 0 the related equation.

Let $p(z, \lambda), q(z, \lambda), a(z, \lambda)$, and $\mathfrak{e}(z, \lambda)$, be polynomials of the degree $m \mathrm{i}$ $1 / \lambda$, and $r(z, \lambda)$ a power series, thus

$$
\begin{gathered}
p(z, \lambda)=\sum_{n=0}^{m} \frac{p_{n}(z)}{\lambda^{n}}, \quad q(z, \lambda)=\sum_{n=0}^{m} \frac{q_{n}(z)}{\lambda^{n}}, \\
r(z, \lambda)=\sum_{n=0}^{\infty} \frac{r_{n}(z)}{\lambda^{n}},
\end{gathered}
$$


and

$$
\mathfrak{Q}(z, \lambda)=\sum_{n=0}^{m} \frac{\alpha_{n}(z)}{\lambda^{n}}, \quad \mathfrak{e}(z, \lambda)=\sum_{n=0}^{m} \frac{\gamma_{n}(z)}{\lambda^{n}},
$$

with coefficients $p_{n}(z), q_{n}(z), r_{n}(z), \alpha_{n}(z)$, and $\gamma_{n}(z)$, that are analytic functions in the domain $R_{z}$, and which, beyond that, are to be determined below. In more explicit form the equation (2.2) is

$$
u^{\mathrm{iv}}+\frac{r}{\lambda^{m+1}} u^{\prime \prime \prime}+\lambda^{2}\left\{p u^{\prime \prime}+\left[p^{\prime}+q+\frac{p r}{\lambda^{m+1}}\right] u^{\prime}\right.
$$

$$
\left.+\left[q^{\prime}+\frac{q r}{\lambda^{m+1}}\right] u\right\}=0 .
$$

With $u$ taken to be any solution of this equation, the relation (2.3) defines a corresponding function $\eta$. The derivative of this $\eta$ is a linear form in $u, u^{\prime}$, $u^{\prime \prime}$, and $u^{\prime \prime \prime}$, and its higher derivatives are likewise representable by such forms, if at each differentiation the function $u^{i v}$ is eliminated by the use of the equation (2.8). We shall need these forms, but we shall need them explicitly only to the extent of their terms in $1 / \lambda^{m}$. Therefore we shall represent them with their terms of degrees higher than the $m$ th in $1 / \lambda$ designated in the aggregate by $E(z, \lambda) / \lambda^{m+1}$. By this the symbol $E(z, \lambda)$ is introduced as a generic designation for a function which is analytic in $z$ and a power series in $1 / \lambda$. To this symbolism we shall hold throughout the paper. In conjunction with (2.3) we have, therefore, the formulas

$$
\begin{aligned}
\eta^{\prime}= & a^{\prime} u+a u^{\prime}+\frac{1}{\lambda^{2}} \mathfrak{e}^{\prime} u^{\prime \prime}+\frac{1}{\lambda^{2}} \mathfrak{e} u^{\prime \prime \prime} \\
\eta^{\prime \prime}= & {\left[Q^{\prime \prime}-q \mathfrak{e}+\frac{1}{\lambda^{m+1}} E\right] u+\left[2{Q^{\prime}}^{\prime}\left(p^{\prime}+q\right) \mathfrak{e}+\frac{1}{\lambda^{m+1}} E\right] u^{\prime} } \\
& +\left[a-p \mathfrak{e}+\frac{1}{\lambda^{2}} \mathfrak{e}^{\prime \prime}\right] u^{\prime \prime}+\left[\frac{2}{\lambda^{2}} \mathfrak{e}^{\prime}+\frac{1}{\lambda^{m+3}} E\right] u^{\prime \prime \prime}, \\
\eta^{\prime \prime \prime}= & {\left[Q^{\prime \prime \prime}-3 q^{\prime} \mathfrak{C}^{\prime}-q^{\prime \prime} \mathfrak{e}+\frac{1}{\lambda^{m+1}} E\right] u } \\
& +\left[3 Q^{\prime \prime}-3\left(p^{\prime}+q\right) \mathfrak{e}^{\prime}-\left(p^{\prime \prime}+2 q^{\prime}\right) \mathfrak{e}+\frac{1}{\lambda^{m+1}} E\right] u^{\prime} \\
& +\left[3 Q^{\prime}-3 p \mathfrak{C}^{\prime}-\left(2 p^{\prime}+q\right) \mathfrak{e}+\frac{1}{\lambda^{2}} \mathfrak{e}^{\prime \prime \prime}+\frac{1}{\lambda^{m+1}} E\right] u^{\prime \prime} \\
& +\left[a-p \mathfrak{e}+\frac{3}{\lambda^{2}} \mathfrak{e}^{\prime \prime}+\frac{1}{\lambda^{m+3}} E\right] u^{\prime \prime \prime},
\end{aligned}
$$


and finally that

$$
\begin{aligned}
\eta^{\mathrm{iv}}= & {\left[-\lambda^{2} q^{\prime}(a-p \mathfrak{e})+a^{\mathrm{iv}}-6 q^{\prime} \mathfrak{e}^{\prime \prime}-4 q^{\prime \prime} \mathfrak{e}^{\prime}-q^{\prime \prime \prime} \mathfrak{e}+\frac{1}{\lambda^{m-1}} E\right] u } \\
& +\left[-\lambda^{2}\left(p^{\prime}+q\right)(a-p \mathfrak{e})+4 Q^{\prime \prime \prime}-6\left(p^{\prime}+q\right) \mathfrak{e}^{\prime \prime}-4\left(p^{\prime \prime}+2 q^{\prime}\right) \mathfrak{e}^{\prime}\right. \\
& +\left[-\lambda^{\prime \prime \prime} p\left(a-p q^{\prime \prime}\right) \mathfrak{e}+\frac{1}{\lambda^{m-1}} E\right] u^{\prime} \\
& \left.\quad+\frac{1}{\lambda^{2}} \mathfrak{e}^{\mathrm{iv}}+\frac{1}{\lambda^{m+1}} E\right] u^{\prime \prime} \\
& +\left[4 a^{\prime}-4 p \mathfrak{C}^{\prime}-\left(3 p^{\prime}+q\right) \mathfrak{e}+\frac{4}{\lambda^{2}} \mathfrak{e}^{\prime \prime \prime}+\frac{1}{\lambda^{m+1}} E\right] u^{\prime \prime \prime} .
\end{aligned}
$$

With the operator $\mathscr{L}$ as defined in (1.2), therefore, it follows that

$$
\mathscr{L}(\eta)=\lambda^{2}\left\{S_{0} u+S_{1} u^{\prime}+S_{2} u^{\prime \prime}\right\}+S_{3} u^{\prime \prime \prime},
$$

with

$$
\begin{aligned}
& S_{0}=P Q^{\prime \prime}+Q Q^{\prime}+\left[R-q^{\prime}\right] a+[p-P] q^{\prime} \mathbb{C} \\
& +\frac{1}{\lambda^{2}}\left\{Q^{\mathrm{iv}}-6 q^{\prime} \mathfrak{C}^{\prime \prime}-4 q^{\prime \prime} \mathfrak{C}^{\prime}-q^{\prime \prime \prime} \mathfrak{C}\right\}+\frac{1}{\lambda^{m+1}} E, \\
& S_{1}=2 P Q^{\prime}-\left[p^{\prime}+q-Q\right] a+[p-P]\left[p^{\prime}+q\right] \mathfrak{e} \\
& +\frac{1}{\lambda^{2}}\left\{4 a^{\prime \prime \prime}-6\left[p^{\prime}+q\right] \mathfrak{e}^{\prime \prime}-4\left[p^{\prime \prime}+2 q^{\prime}\right] \mathfrak{e}^{\prime}-\left[p^{\prime \prime \prime}+3 q^{\prime \prime}\right] \mathfrak{e}\right\} \\
& +\frac{1}{\lambda^{m+1}} E, \\
& S_{2}=[P-p][a-p \mathfrak{e}]+\frac{1}{\lambda^{2}}\left\{6 \mathfrak{a}^{\prime \prime}-[6 p-P] \mathfrak{e}^{\prime \prime}-\left[8 p^{\prime}+4 q-Q\right] \mathfrak{e}^{\prime}\right. \\
& \left.-\left[3 p^{\prime \prime}+3 q^{\prime}-R\right] \mathfrak{e}\right\}+\frac{1}{\lambda^{4}} \mathfrak{e}^{\mathrm{iv}}+\frac{1}{\lambda^{m+3}} E, \\
& S_{3}=[2 P-4 p] \mathfrak{e}^{\prime}-\left[3 p^{\prime}+q-Q\right] \mathfrak{e}+4 Q^{\prime}+\frac{4}{\lambda^{2}} \mathfrak{e}^{\prime \prime \prime}+\frac{1}{\lambda^{m+1}} E .
\end{aligned}
$$

The functions $S_{j}$ as thus given are all expressible as power series in $1 / \lambda$. We propose to remove from these series their initial polynomial segments of degree $m$, by making appropriate determinations of the hitherto unspecified coefficients of the formulas (2.5) and (2.7). 
3. The first determinations. The constant terms of the formulas (2.11) for the functions $S_{j}(z, \lambda)$ in powers of $1 / \lambda$, are the left-hand members of the equations

$$
\begin{aligned}
P_{0} \alpha_{0}^{\prime \prime}+Q_{0} \alpha_{0}^{\prime}+R_{0} \alpha_{0}-\alpha_{0} q_{0}^{\prime}+\left[p_{0}-P_{0}\right] q_{0}^{\prime} \gamma_{0}=0 \\
2 P_{0} \alpha_{0}^{\prime}-p_{0}^{\prime} \alpha_{0}+Q_{0} \alpha_{0}-\alpha_{0} q_{0}+\left[p_{0}-P_{0}\right]\left[p_{0}^{\prime}+q_{0}\right] \gamma_{0}=0 \\
{\left[P_{0}-p_{0}\right]\left[\alpha_{0}-p_{0} \gamma_{0}\right]=0 } \\
{\left[2 P_{0}-4 p_{0}\right] \gamma_{0}^{\prime}-\left[3 p_{0}^{\prime}+q_{0}-Q_{0}\right] \gamma_{0}+4 \alpha_{0}^{\prime}=0 . }
\end{aligned}
$$

These equations constitute a nonlinear simultaneous differential system for the functions $p_{0}(z), q_{0}(z), \alpha_{0}(z)$ and $\gamma_{0}(z)$. We shall show that the system admits a solution, the elements of which are analytic functions. By assigning these solution values to the functions, we shall achieve the removal of the initial terms of the formulas (2.11).

The third one of the equations (3.1) is solved at once by taking

$$
p_{0}(z) \equiv P_{0}(z) \text {. }
$$

In consequence of this, the first two equations (3.1) involve as unknowns only the functions $q_{0}(z)$ and $\alpha_{0}(z)$. On adding to this pair the derivative of the second equation, we obtain a system from which the quantities $q_{0}(z)$ and $q_{0}^{\prime}(z)$ can be eliminated. The eliminant relation can, by virtue of $(3.2)$, be written in the form

$$
P_{0}\left(\frac{1}{\alpha_{0}}\right)^{\prime \prime}+\left[2 P_{0}^{\prime}-Q_{0}\right]\left(\frac{1}{\alpha_{0}}\right)^{\prime}+\left[P_{0}^{\prime \prime}-Q_{0}^{\prime}+R_{0}\right]\left(\frac{1}{\alpha_{0}}\right)=0 .
$$

This is a differential equation for $1 / \alpha_{0}(z)$.

The equation (3.3) is singular at the origin. Since it is, in fact, the adjoint of the differential equation (1.7), its exponents relative to $z=0$ are 0 and $-\rho$. Because $\rho$ is not a negative integer, by hypothesis, the solution to which the exponent 0 applies is analytic, namely nonlogarithmic. To its constant term we may assign the value 1 . Therewith $1 / \alpha_{0}(z)$, and hence $\alpha_{0}(z)$ itself, have been determined so that

$$
\alpha_{0}(0)=1 \text {, }
$$

and the determinations are analytic in $R_{z}$ when that region is suitably confined.

The second equation (3.1) now gives for $q_{0}(z)$ the formula

$$
q_{0}(z)=Q_{0}(z)-P_{0}^{\prime}(z)+2 P_{0}(z) \frac{\alpha_{0}^{\prime}(z)}{\alpha_{0}(z)},
$$

whereas, with this at hand, the final equation (3.1) is a differential equation for $\gamma_{0}(z)$. This can be written in the form 


$$
\gamma_{0}^{\prime}+\left[\frac{P_{0}^{\prime}}{P_{0}}+\frac{\alpha_{0}^{\prime}}{\alpha_{0}}\right] \gamma_{0}=\frac{2 \alpha_{0}^{\prime}}{P_{0}},
$$

and accordingly has the integral

$$
\gamma_{0}(z)=\frac{1}{P_{0}(z)}\left[\alpha_{0}(z)-\frac{1}{\alpha_{0}(z)}\right] .
$$

There is an indeterminacy in this formula at the origin. However, if $\gamma_{0}(z)$ is taken to be continuous, its determination is thereby made analytic. It is useful to observe that the relation

$$
\left[\alpha_{0}(z)-p_{0}(z) \gamma_{0}(z)\right] \neq 0
$$

is assured for all $z$ in $R_{\mathbf{z}}$. This follows from the fact that the left-hand member of the relation has, by (3.6) and (3.2), the value $1 / \alpha_{0}(z)$.

4. The second determinations. The coefficients of $1 / \lambda$ in the formulas (2.11) are the left-hand members of the respective equations

$$
\begin{array}{r}
P_{0} \alpha_{1}^{\prime \prime}+Q_{0} \alpha_{1}^{\prime}+\left[R_{0}-q_{0}^{\prime}\right] \alpha_{1}-\alpha_{0} q_{1}^{\prime}+f_{0,1}=0, \\
2 P_{0} \alpha_{1}^{\prime}-\left[p_{0}^{\prime}+q_{0}-Q_{0}\right] \alpha_{1}-\alpha_{0} q_{1}+f_{1,1}=0, \\
{\left[P_{1}-p_{1}\right]\left[\alpha_{0}-p_{0} \gamma_{0}\right]=0,} \\
-2 P_{0} \gamma_{1}^{\prime}-\left[3 p_{0}^{\prime}+q_{0}-Q_{0}\right] \gamma_{1}+f_{3,1}=0,
\end{array}
$$

in which

$$
\begin{aligned}
& f_{0,1}=P_{1} \alpha_{0}^{\prime \prime}+Q_{1} \alpha_{0}^{\prime}+R_{1} \alpha_{0}+\left[p_{1}-P_{1}\right] q_{0}^{\prime} \gamma_{0}, \\
& f_{1,1}=2 P_{1} \alpha_{0}^{\prime}-\left[p_{1}^{\prime}-Q_{1}\right] \alpha_{0}+\left[p_{1}-P_{1}\right]\left[p_{0}^{\prime}+q_{0}\right] \gamma_{0}, \\
& f_{3,1}=4 \alpha_{1}^{\prime}+\left[2 P_{1}-4 p_{1}\right] \gamma_{0}^{\prime}-\left[3 p_{1}^{\prime}+q_{1}-Q_{1}\right] \gamma_{0} .
\end{aligned}
$$

We shall show that the functions $p_{1}(z), q_{1}(z), \alpha_{1}(z)$ and $\gamma_{1}(z)$ can be determined to be analytic and to fulfill these equations. By making those determinations we shall remove the terms in $1 / \lambda$ from the formulas $(2.11)$. One special case can'be quickly disposed of. If the differential equation (1.1) does not involve the first power of $\lambda$, namely if $P_{1}(z), Q_{1}(z)$ and $R_{1}(z)$ are all zero, the system (4.1) is fulfilled by the determinations

$$
p_{1}(z) \equiv 0, \quad q_{1}(z) \equiv 0, \quad \alpha_{1}(z) \equiv 0, \quad \gamma_{1}(z) \equiv 0 .
$$

In the general case the discussion proceeds as follows.

Because of the relation (3.7), the third equation (4.1) imposes the determination

$$
p_{1}(z) \equiv P_{1}(z) \text {. }
$$

This makes the functions $f_{0,1}$ and $f_{1,1}$ known, and the first two equations (4.1) are accordingly a system for the functions $a_{1}(z)$ and $\alpha_{1}(z)$. We deal with this 
system in the following way. Let the variable $I_{1}$ be introduced to replace $\alpha_{1}$ by means of the relation

$$
\alpha_{1}=\alpha_{0} \int \frac{I_{1}(z)}{\alpha_{0}(z)} d z
$$

Because $\alpha_{0}$ fulfills the first two equations (3.1), this transforms the initial pair of equations (4.1) into the forms

$$
\begin{array}{r}
P_{0} I_{1}^{\prime}+\left[P_{0} \frac{\alpha_{0}^{\prime}}{\alpha_{0}}+Q_{0}\right] I_{1}-\alpha_{0} q_{1}^{\prime}+f_{0,1}=0, \\
2 P_{0} I_{1}-\alpha_{0} q_{1}+f_{1,1}=0 .
\end{array}
$$

To this pair of equations we add the derivative of the second one, and from the resulting system of three equations eliminate the functions $q_{1}(z)$ and $q_{1}^{\prime}(z)$. The eliminant relation is

$$
P_{0} I_{1}^{\prime}+\left[2 P_{0}^{\prime}-Q_{0}-3 P_{0} \frac{\alpha_{0}^{\prime}}{\alpha_{0}}\right] I_{1}=\left[f_{0,1}-f_{1,1}^{\prime}+f_{1,1} \frac{\alpha_{0}^{\prime}}{\alpha_{0}}\right] \text {. }
$$

This is a differential equation for $I_{1}(z)$. It has a singular point at $z=0$, and, with respect to this, the exponent $-\rho-1$. Since $\rho$ is not a negative integer, this exponent is neither zero nor a positive integer, and the equation (4.7), being nonhomogeneous, accordingly admits an analytic solution. We take this solution for $I_{1}(z)$. In terms of this the second equation (4.6) gives $q_{1}(z)$ by the formula

$$
q_{1}(z)=\frac{1}{\alpha_{0}(z)}\left[f_{1,1}(z)+2 P_{0}(z) I_{1}(z)\right]
$$

whereas $\alpha_{1}(z)$ is given by the relation (4.5).

With the determination of $\alpha_{1}(z)$ thus made, the function $f_{3,1}$ has become known, and the fourth equation (4.1) is accordingly one in which $\gamma_{1}(z)$ is the only unknown. We may give this equation the form

$$
\gamma_{1}^{\prime}+\left[\frac{P_{0}^{\prime}}{P_{0}}+\frac{\alpha_{0}^{\prime}}{\alpha_{0}}\right] \gamma_{1}=\frac{f_{3,1}}{2 P_{0}} .
$$

It accordingly has the integral

$$
\gamma_{1}(z)=\frac{1}{2 P_{0} \alpha_{0}} \int_{0}^{z} \alpha_{0}(z) f_{3,1}(z) d z .
$$

With proper definition at its point of indeterminacy, this is analytic.

5. Further determinations. When $n$ is any integer not exceeding $m$, the coefficients of the terms in $1 / \lambda^{n}$ in the formulas (2.11) are the left-hand members of the equations 


$$
\begin{array}{r}
P_{0} \alpha_{n}^{\prime \prime}+Q_{0} \alpha_{n}^{\prime}+\left[R_{0}-q_{0}^{\prime}\right] \alpha_{n}-\alpha_{0} q_{n}^{\prime}+f_{0, n}=0, \\
2 P_{0} \alpha_{n}^{\prime}-\left[p_{0}^{\prime}+q_{0}-Q_{0}\right] \alpha_{n}-\alpha_{0} q_{n}+f_{1, n}=0, \\
{\left[P_{n}-p_{n}\right]\left[\alpha_{0}-p_{0} \gamma_{0}\right]+f_{2, n}=0,} \\
-2 P_{0} \gamma_{n}^{\prime}-\left[3 p_{0}^{\prime}+q_{0}-Q_{0}\right] \gamma_{n}+f_{3, n}=0,
\end{array}
$$

with functions $f_{i, n}(z)$ that are given by the formulas

$$
\begin{aligned}
& f_{0, n}=P_{n} \alpha_{0}^{\prime \prime}+Q_{n} \alpha_{0}^{\prime}+R_{n} \alpha_{0}+\alpha_{n-2}^{\text {iv }}+\sum_{j=2}^{n} \sum_{i=0}^{n-j}\left[p_{i}-P_{j}\right] q_{i}^{\prime} \gamma_{n-i-i} \\
& +\sum_{j=1}^{n-1}\left\{P_{j} \alpha_{n-j}^{\prime \prime}+Q_{j} \alpha_{n-j}^{\prime}+\left[R_{j}-q_{j}^{\prime}\right] \alpha_{n-j}-6 q_{j-1}^{\prime} \gamma_{n-1-j}^{\prime \prime}\right. \\
& \left.-4 q_{j-1}^{\prime \prime} \gamma_{n-j-1}^{\prime}-q_{j-1}^{\prime \prime \prime} \gamma_{n-j-1}\right\}, \\
& f_{1, n}=2 P_{n} \alpha_{0}^{\prime}-\left[p_{n}^{\prime}-Q_{n}\right] \alpha_{0}+4 \alpha_{n-2}^{\prime \prime \prime}+\sum_{j=2}^{n} \sum_{i=0}^{n-i}\left[p_{j}-P_{j}\right]\left[p_{i}^{\prime}+q_{i}\right] \gamma_{n-1-j} \\
& +\sum_{j=1}^{n-1}\left\{2 P_{j} \alpha_{n-j}^{\prime}-\left[p_{j}^{\prime}+q_{j}-Q_{j}\right] \alpha_{n-j}-6\left[p_{j-1}^{\prime}+q_{j-1}\right] \gamma_{n-1-j}^{\prime \prime}\right. \\
& \left.-4\left[p_{j-1}^{\prime \prime}+2 q_{j-1}^{\prime}\right] \gamma_{n-1-j}^{\prime}-\left[p_{j-1}^{\prime \prime \prime}+3 q_{j-1}^{\prime \prime}\right] \gamma_{n-1-j}\right\}, \\
& f_{2, n}=6 \alpha_{n-2}^{\prime \prime}+\gamma_{n-4}^{\mathrm{iv}}-\sum_{j=2}^{n-1} \sum_{i=0}^{n-j}\left[P_{j}-p_{j}\right] p_{i} \gamma_{n-j-i} \\
& +\sum_{j=1}^{n-1}\left\{\left[P_{j}-p_{j}\right] \alpha_{n-j}-\left[6 p_{j-1}-P_{j-1}\right] \gamma_{n-1-j}^{\prime \prime}\right. \\
& \left.-\left[8 p_{j-1}^{\prime}+4 q_{j-1}-Q_{j-1}\right] \gamma_{n-1-j}^{\prime}-\left[3 p_{j-1}^{\prime \prime}+3 q_{j-1}^{\prime}-R_{j-1}\right] \gamma_{n-1-j}\right\}, \\
& f_{3, n}=4 \alpha_{n}^{\prime}+4 \gamma_{n-2}^{\prime \prime \prime}+\sum_{j=1}^{n}\left\{\left[2 P_{j}-4 p_{j}\right] \gamma_{n-j}^{\prime}-\left[3 p_{j}^{\prime}+q_{j}-Q_{j}\right] \gamma_{n-j}\right\} .
\end{aligned}
$$

We shall show, by the method of induction, that the functions $p_{n}(z), q_{n}(z)$, $\alpha_{n}(z)$ and $\gamma_{n}(z)$ can be determined to be analytic, and to fulfill these equations, and thus that all terms in $1 / \lambda^{n}$, with $n \leqq m$, can be removed from the formulas (2.11).

Suppose, therefore, that the functions

$$
p_{j}(z), \quad q_{j}(z), \quad \alpha_{j}(z), \quad \gamma_{j}(z),
$$

for $j=0,1, \cdots,(n-1)$, have been determined and are analytic. The function $f_{2, n}$, as given by (5.2), is then constructed of known analytic functions, and from the third equation (5.1) we draw the determination

$$
p_{n}(z)=P_{n}(z)+\alpha_{0}(z) f_{2, n}(z) .
$$

In consequence of this, the functions $f_{0, n}$ and $f_{1, n}$ are known, and the first two 
equations (5.1) are accordingly a system for the functions $q_{n}(z)$ and $\alpha_{n}(z)$. The substitution

$$
\alpha_{n}=\alpha_{0} \int \frac{I_{n}(z)}{\alpha_{0}(z)} d z
$$

transforms this system into

$$
\begin{array}{r}
P_{0} I_{n}^{\prime}+\left[P_{0} \frac{\alpha_{0}^{\prime}}{\alpha_{0}}+Q_{0}\right] I_{n}-\alpha_{0} q_{n}^{\prime}+f_{0, n}=0, \\
2 P_{0} I_{n}-\alpha_{0} q_{n}+f_{1, n}=0 .
\end{array}
$$

When this new system has been augmented by adjoining the derivative of the second of its equations to it, the elimination of $q_{n}(z)$ and $q_{n}^{\prime}(z)$ is possible, and results in the equation

$$
P_{0} I_{n}^{\prime}+\left[2 P_{0}^{\prime}-Q_{0}-3 P_{0} \frac{\alpha_{0}^{\prime}}{\alpha_{0}}\right] I_{n}=\left[f_{0, n}-f_{1, n}^{\prime}+f_{1, n} \frac{\alpha_{0}^{\prime}}{\alpha_{0}}\right] .
$$

This equation has an analytic solution, which we take for $I_{n}(z)$. The second equation (5.6) then yields the formula

$$
q_{n}(z)=\frac{1}{\alpha_{0}(z)}\left[f_{1, n}(z)+2 P_{0}(z) I_{n}(z)\right]
$$

and the formula (5.5) gives $\alpha_{n}(z)$.

The function $f_{3, n}$ of (5.2) is now known. The fourth equation (5.1), which can be written in the form

$$
\gamma_{n}^{\prime}+\left[\frac{P_{0}^{\prime}}{P_{0}}+\frac{\alpha_{0}^{\prime}}{\alpha_{0}}\right] \gamma_{n}=\frac{f_{3, n}}{2 P_{0}},
$$

is accordingly a differential equation for $\gamma_{n}(z)$. It has the integral

$$
\gamma_{n}(z)=\frac{1}{2 P_{0} \alpha_{0}} \int_{0}^{z} \alpha_{0}(z) f_{3, n}(z) d z .
$$

This is what was to be shown.

It is worth observing that for an equation (1.1) that involves only even powers of $\lambda$ the functions (5.3) with odd subscripts are all identically zero. This should, of course, be expected. It is easily proved by the method of induction, for it will be seen that if the functions (5.3) vanish for odd values of $j$ that are less than $n$, and $n$ itself is odd, then the functions (5.2) are all zero.

6. The differential equation in $\eta$. With the functions $p(z, \lambda), q(z, \lambda)$, $a(z, \lambda)$, and $\mathfrak{e}(z, \lambda)$ now determined, the coefficients of the differential equation (2.2) are specified except for terms that are of higner degree in $1 / \lambda$ than the $m$ th. Also the formula (2.3) has been made specific. Through this formula 
each solution of the equation (2.2) defines a corresponding function $\eta$. These latter functions are, in their turn, the solutions of a certain differential equation. This equation we are now ready to determine.

From the system of five equations (2.3), (2.9) and (2.10) we may eliminate the quantities $u, u^{\prime}, u^{\prime \prime}$ and $u^{\prime \prime \prime}$. The eliminant relation, which is the equation we wish to obtain, is found to be

$$
D_{1}(z, \lambda) \eta-D_{2}(z, \lambda) \eta^{\prime}+D_{3}(z, \lambda) \eta^{\prime \prime}-D_{4}(z, \lambda) \eta^{\prime \prime \prime}+D_{5}(z, \lambda) \&(\eta)=0,
$$

in which the coefficient $D_{i}(z, \lambda)$, for any $i$, is the determinant that results from the elision of the elements of the $i$ th row from the array

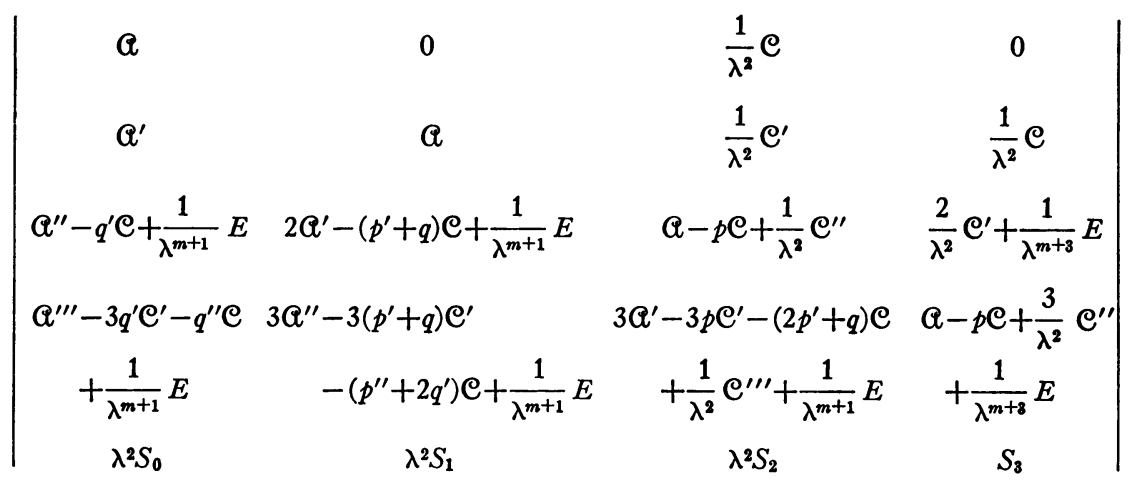

The coefficient $D_{i}(z, \lambda)$ with $i=5$ does not involves the function $S_{j}$. It can evidently be expressed as a power series in $1 / \lambda$, and the leading term of that series is found to be $\alpha_{0}^{2}\left[\alpha_{0}-p_{0} \gamma_{0}\right]^{2}$. By (3.6) and (3.2) the value of this is identically 1 . This coefficient $D_{5}(z, \lambda)$ is therefore nonvanishing in $R_{z}$ when $|\lambda|$ is sufficiently large, and its reciprocal, $D_{5}^{-1}(z, \lambda)$ is likewise expressible in powers of $1 / \lambda$ with 1 as the constant term. We may therefore write the equation (6.1) in the form

$$
\mathfrak{L}(\eta)+l(\eta, z)=0
$$

with

$$
l(\eta, z)=-D_{5}^{-1}\left[D_{4} \eta^{\prime \prime \prime}-D_{3} \eta^{\prime \prime}+D_{2} \eta^{\prime}-D_{1} \eta\right] .
$$

The coefficients $D_{i}(z, \lambda)$, with $i=1,2,3,4$, all do depend upon the functions $S_{j}$, and these latter were reduced to be of the order of $1 / \lambda^{m+1}$ by the determinations of the functions (2.5) and (2.7). From their formulas it is therefore easily seen that these coefficients with $i=1,2,3$ are of the order of $1 / \lambda^{m-1}$, whereas $D_{4}$ is of the order of $1 / \lambda^{m+1}$. The structure of the formula (6.4) is thus

$$
l(\eta, z)=\lambda^{-m-1}\left[E(z, \lambda) \eta^{\prime \prime \prime}+\lambda^{2} E(z, \lambda) \eta^{\prime \prime}+\lambda^{2} E(z, \lambda) \eta^{\prime}+\lambda^{2} E(z, \lambda) \eta\right] .
$$

We make one further observation concerning the coefficient $D_{5}(z, \lambda)$. Let 
$u_{0}(z), u_{1}(z), u_{2}(z), u_{3}(z)$, be any linearly independent solutions of the differential equation (2.8), and let their Wronskian, as a function of $z$, be denoted by $W\left(u_{0}, u_{1}, u_{2}, u_{3} ; z\right)$. The equations (2.3) and (2.9) then show that the Wronskian of the corresponding functions $\eta$ is given by the formula

$$
W\left(\eta_{0}, \eta_{1}, \eta_{2}, \eta_{3} ; z\right)=D_{5}(z, \lambda) W\left(u_{0}, u_{1}, u_{2}, u_{3} ; z\right) \text {. }
$$

From this it follows that

$$
\frac{D_{5}^{\prime}(z, \lambda)}{D_{5}(z, \lambda)}=\frac{W^{\prime}\left(\eta_{0}, \eta_{1}, \eta_{2}, \eta_{3} ; z\right)}{W\left(\eta_{0}, \eta_{1}, \eta_{2}, \eta_{3} ; z\right)}-\frac{W^{\prime}\left(u_{0}, u_{1}, u_{2}, u_{3} ; z\right)}{W\left(u_{0}, u_{1}, u_{2}, u_{3} ; z\right)} .
$$

Now the terms on the right of this equation have respectively the values of the coefficients of $\eta^{\prime \prime \prime}$ and $u^{\prime \prime \prime}$ in the differential equations (2.8) and (6.3). Since these coefficients are each of the order of $1 / \lambda^{m+1}$, it follows that $D_{5}^{\prime} / D_{5}$ is of that order, and hence that $D_{5}^{\prime}(z, \lambda)$ is itself so. The inference which this permits is, that in the expression of $D_{5}(z, \lambda)$ as a power series in $1 / \lambda$, the terms, to those of the $m$ th degree, are all constants with respect to $z$.

A summary of the formal deductions we have thus far made is the following. We have determined the functions (2.5), and therewith the differential equation (2.4). This is a differential equation of the third order, of a type for which the forms of complete sets of linearly independent solutions are known [9]. These solutions are, however, clearly also solutions of the equation (2.2), namely of (2.8), and their corresponding functions $\eta$ given by the formula (2.3), are therefore solutions of the differential equation (6.3). To complete the set of solutions for this latter equation, one more solution is requisite. We shall determine such a one by an appropriate specification of the function $r(z, \lambda) / \lambda^{m+1}$ of (2.6). This latter function, since it is of the order of $1 / \lambda^{m+1}$ has played no role in our deductions thus far.

The equation (6.3) has coefficients which differ from those of the given differential equation (1.1) only by terms that are of the relative order of $1 / \lambda^{m+1}$. When we have made it solvable by our choice of the function $r(z, \lambda)$ namely when we have made it an equation whose solution forms are all known, we shall call it the differential equation related to the given one.

\section{PART II. The General THEORY}

7. The determination of $r(z, \lambda)$. Differential equations of "regular" type. Since the solutions of the equation (2.4) are known, and are also solutions of the differential equation (2.2), we have available three linearly independent solutions of this latter equation. To make up a complete set of solutions another one must be obtained. We shall now see that such may be done, at least generally, by a determination of the coefficient $r(z, \lambda)$ in the equation (2.2). Inasmuch as this coefficient appears in the equation only in product with $1 / \lambda^{m+1}$, it has not been of sufficiently low degree in $1 / \lambda$ to have affected the deductions thus far. 
Let $u_{3}(z, \lambda)$ be the polynomial of degree $m$ in $1 / \lambda$,

$$
u_{3}(z, \lambda)=\sum_{n=0}^{m} \frac{U_{n}(z)}{\lambda^{n}}
$$

in which the coefficients $U_{n}(z)$ are analytic but undetermined. From the formula (2.1), with $p(z, \lambda)$ and $q(z, \lambda)$ given by (2.5), we find, then, the evalua$\operatorname{tion}\left({ }^{2}\right)$

$$
L\left(u_{3}\right)=\lambda^{2} \sum_{n=0}^{2 m \prime} \frac{p_{0} U_{n}^{\prime}+q_{0} U_{n}+H_{n}(U)}{\lambda^{n}},
$$

where $2 m^{\prime}$ is the larger one of the integers $2 m$, and $m+2$, and

$$
H_{n}(U) \equiv U_{n-2}^{\prime \prime \prime}+\sum_{j=1}^{n}\left[p_{j} U_{n-j}^{\prime}+q_{j} U_{n-j}\right] .
$$

We propose to determine the functions $U_{n}(z)$ so as to give to the leading term of the sum in (7.2) the value 1 , and to each one of the next following $m$ terms the value 0 .

Since $H_{0}(U) \equiv 0$, the leading term of the sum in (7.2) will have the value 1 if

$$
p_{0} U_{0}^{\prime}+q_{0} U_{0}=1 .
$$

This differential equation for $U_{0}(z)$ has a singular point at $z=0$. Its exponent there is seen directly to be $-q_{0}(0) / p_{0}^{\prime}(0)$, and, by virtue of the determinations (3.2) and (3.5), this is precisely the constant $\rho$ that is given by the formula (1.9). Now whether or not the differential equation (7.4) admits of an analytic solution, is familiarly bound up with this exponent $\rho$, specifically with whether $\rho$ is or is not zero or a positive integer. We shall distinguish between these cases, and shall designate the given differential equation (1.1) as being of the regular type if $\rho$ is not zero or a positive integer, and as of an irregular type in the contrary case. The discussion of differential equations of an irregular type we shall relegate to Part III of this paper. Throughout the present part, namely Part II, we shall therefore confine the attention to differential equations of the regular type. For these the differential equation (7.4) admits of an analytic solution. We take this for $U_{0}(z)$.

The $m$ terms after the first one of the sum in (7.2) vanish if

$$
p_{0} U_{n}^{\prime}+q_{0} U_{n}=-H_{n}(U), \quad n=1,2, \cdots, m .
$$

It is easily shown, by induction, that the functions $U_{n}(z)$ may be determined to be analytic and to fulfill these equations. The function $H_{n}(z)$ is known when the functions $U_{j}(z)$ for $j<n$ have been determined. The equation (7.5)

(2) It is to be understood, of course, that any symbol $p, q$, or $U$, with a subscript that does not occur in (2.1) or (7.1) is to be assigned the value 0 . 
is then a differential equation for $U_{n}(z)$. Like the equation (7.4) it admits of an analytic solution. We give $U_{n}(z)$ that determination for $n=1,2, \cdots, m$. The consequence of this is that the evaluation (7.2) has been made to be more specifically

$$
L\left(u_{3}\right)=\lambda^{2}\left[1+\sum_{n=m+1}^{2 m^{\prime}} \frac{H_{n}(U)}{\lambda^{n}}\right] .
$$

By virtue of (7.6) the function $L\left(u_{3}\right)$ is of the order of $\lambda^{2}$ and its derivative is of the order of $\lambda^{-m+1}$. Therefore the ratio $-L^{\prime}\left(u_{3}\right) / L\left(u_{3}\right)$ is of the order of $1 / \lambda^{m+1}$, and the product of $\lambda^{m+1}$ by this ratio is a power series in $1 / \lambda$. We assign this series to be $r(z, \lambda)$, thus

$$
r(z, \lambda)=-\lambda^{m+1} \frac{L^{\prime}\left(u_{3}\right)}{L\left(u_{3}\right)} .
$$

Now with this determination it is clear that $u_{3}(z, \lambda)$ is a solution of the differential equation (2.2). Were it to be linearly dependent upon the solutions which also fulfill the equation (2.4), it would itself fulfill this latter equation. Since it fulfills the relation (7.6), which is contradictory to (2.4), no such linear dependence maintains. The function $u_{3}(z, \lambda)$ and any three linearly independent solutions of the equation (6.7) accordingly make up a complete set of solutions of the equation (2.2). The corresponding functions $\eta$, under the relation (2.3), thus comprise a fundamental set of solutions of the differential equation (6.3). This latter equation is therefore solvable, in the sense that its solution forms are known. We shall henceforth refer to it as the related equation.

8. Solutions of the related equation. Of the solutions of the related equation (6.3), one, namely the one that corresponds, through the formula (2.3), to $u_{3}(z, \lambda)$ as given by $(7.1)$, is explicitly known. The others correspond similarly to solutions of the equation (2.4). Concerning these latter the following facts are known [9].

The relation

$$
\xi=\frac{i}{2} \lambda \int_{0}^{z} p_{0}^{1 / 2}(z) d z
$$

defines $\xi$ as a variable that depends upon $\lambda$ and $z$. Near $z=0$ this variable has the character of $\lambda z^{3 / 2}$, and is thus double valued. To account for this consistently the domain $R_{z}$ will be taken henceforth to be a two-sheeted Riemann surface with $z=0$ as a branch point, and the only one. The inverse of $\xi$ as a function of $z$ is three-valued. Therefore the image of $R_{z}$ under the mapping relation $(8.1)$ is a three-sheeted Riemann surface $R_{\xi}$. Upon this again the origin is a branch point, and is the only one. The domain $R_{\xi}$ depends upon $\lambda$ as well as upon $R_{z}$. This dependence, however, applies only to its orientation 
and size; - by changes in $\arg \lambda$ it is rotated about its origin, and by increases in $|\lambda|$ it is proportionately magnified.

Any chosen points $\lambda$ and $z$, of which $\lambda \neq 0$ and $z$ is in $R_{z}$, are included in the regions of a so-called associated pair. Of such a pair of regions, the one, $\Lambda$, is a sector of the complex $\lambda$-plane with vertex at the origin, and the other, $R_{z}^{\Lambda}$, is a sub-region of $R_{z}$ that in every case includes the origin [9, p. 104]. Every associated pair of regions has an integer $h$ attached to it. This is by virtue of the fact that $R_{\xi}^{\Lambda}$, the image of $R_{z}^{\Lambda}$ under the mapping (8.1), is, for every $\lambda$ in $\Lambda$, wholly within the sector

$$
\left(h-\frac{3}{2}\right) \pi+\epsilon \leqq \arg \xi \leqq\left(h+\frac{1}{2}\right) \pi-\epsilon,
$$

$\epsilon$ being a suitably small positive constant. The differential equation (2.4) has a set of solutions $u_{h, j}(z, \lambda), j=0,1,2$, which for all $\lambda$ and $z$ in these regions $\Lambda$, $R_{z}^{\Lambda}$ have the forms

$$
\begin{aligned}
\frac{d^{k} u_{h, 0}}{d z^{k}} & =\frac{d^{k} y_{h, 0}}{d z^{k}}+\frac{\zeta^{2 \mu+k / 3} E(z, \lambda) \log \lambda}{\lambda^{m_{1}+1-2 k / 3}}, \\
\frac{d^{k} u_{h, 1}}{d z^{k}} & =\frac{d^{k} y_{h, 1}}{d z^{k}}+\frac{\zeta^{\mu-1 / 2+k / 3} e^{2 \xi} E(z, \lambda) \log \lambda}{\lambda^{m_{1}+1-2 k / 3}}, \\
\frac{d^{k} u_{h, 2}}{d z^{k}} & =\frac{d^{k} y_{h, 2}}{d z^{k}}+\frac{\zeta^{\mu-1 / 2+k / 3} e^{-2 \xi} E(z, \lambda) \log \lambda}{\lambda^{m_{1}+1-2 k / 3}} .
\end{aligned}
$$

We proceed to explain these formulas.

The integer $m_{1}$, which is denoted by $m$ in [9] but which is not necessarily the $m$ of the present paper, may be chosen arbitrarily large. For our present purpose we shall take it to be the larger one of the integers $m$ and 3 .

The functions $y_{h, j}$ are expressed in terms of certain other functions $V_{h, j}$ thus

$$
y_{h, j}(z, \lambda)=A(z, \lambda) V_{h, j}+\frac{1}{\lambda} B(z, \lambda) V_{h, j}^{\prime}+\frac{1}{\lambda^{2}} C(z, \lambda) V_{h, j}^{\prime \prime}, \quad j=0,1,2,
$$

with coefficients $A(z, \lambda), B(z, \lambda)$ and $C(z, \lambda)$ that are specifically determinable polynomials of degree $m_{1}$ in $1 / \lambda$, having coefficients that are analytic in $z$ $[9, \S 3]$. Of the functions $V_{h, j}$ the first one is given by the formula

$$
V_{h, 0}(z, \lambda)=\frac{3^{3 \mu-1} e^{(1-2 h) \mu \pi i}}{\Gamma(3 \mu)} \Psi(z) \sum_{n=0}^{\infty} \frac{\Gamma(\mu+n / 3)}{\Gamma(n+1)}\left[3 e^{-(2 \pi i / 3) h \xi^{2 / 3}}\right]^{n}
$$

The other two are given by the formulas 


$$
\begin{aligned}
& 2\left(\frac{\pi}{3}\right)^{1 / 2} e^{(h-1)(\mu-1 / 2) \pi i} \Psi(z) \sum_{n=0}^{\infty} \frac{\left[3 e^{(2 \pi i / 3)(h-1)} \xi^{2 / 3}\right]^{n}}{\Gamma(n+1) \Gamma(1-\mu-n / 3)}, \\
& 2\left(\frac{\pi}{3}\right)^{1 / 2} e^{h(\mu-1 / 2) \pi i} \Psi(z) \sum_{n=0}^{\infty} \frac{\left[3 e^{(2 \pi i / 3) h} \xi^{2 / 3}\right]^{n}}{\Gamma(n+1) \Gamma(1-\mu-n / 3)},
\end{aligned}
$$

in the order $V_{h, 1}(z, \lambda)$ and $V_{h, 2}(z, \lambda)$ respectively if the integer $h$ is even, and in the order $V_{h, 2}(z, \lambda)$ and $V_{h, 1}(z, \lambda)$ respectively if $h$ is odd. The function $\Psi(z)$ that appears here is given by the formula

$$
\Psi(z)=\frac{\left(\frac{2}{3} \int_{0}^{z} p_{0}^{1 / 2}(z) d z\right)^{1 / 3}}{p_{0}^{1 / 2}(z)},
$$

the indeterminacy at $z=0$ being resolved to make it continuous. By virtue of the hypothesis (ii) of $\S 1$ both $\Psi(z)$ and its reciprocal are analytic. Therefore they are, of course, also both nonvanishing in $R_{\mathbf{z}}$. The symbol $\mu$ stands for a certain polynomial of degree $m$ in $1 / \lambda$ with constant coefficients,

$$
\mu=\sum_{n=0}^{m_{1}} \frac{\mu_{n}}{\lambda^{n}}
$$

The leading term of this has the value $q_{0}(0) / 3 p_{0}^{\prime}(0)$, namely, by (3.2), (3.5) and (1.9)

$$
\mu_{0}=-\frac{\rho}{3} .
$$

For suitably large values of $\xi$, namely when $|\xi|>N$, with some sufficiently large positive constant $N$, the functions $V_{h, j}(z, \lambda)$ are representable by the asymptotic formulas

$$
\begin{aligned}
& V_{h, 0}(z, \lambda) \sim \Psi(z) \xi^{-2 \mu} \sum_{n=0}^{\infty} \frac{\kappa_{n}}{[3 i \xi]^{2 n}}, \\
& V_{h, 1}(z, \lambda) \sim \Psi(z) \xi^{\mu-1 / 2} e^{2 \xi} \sum_{n=0}^{\infty} \frac{\nu_{n}}{[-3 i \xi]^{n}}, \\
& V_{h, 2}(z, \lambda) \sim \Psi(z) \xi^{\mu-1 / 2} e^{-2 \xi} \sum_{n=0}^{\infty} \frac{\nu_{n}}{[3 i \xi]^{n}} .
\end{aligned}
$$

The coefficients $\kappa_{n}$ and $\nu_{n}$ in these relations are constants for which formulas are available $[8,(3.5),(3.7)]$. Finally, in the formulas (8.3) the symbol $\zeta$ is to be interpreted thus

$$
\zeta=\left\{\begin{array}{lll}
N, & \text { when } & |\xi| \leqq N \\
\xi, & \text { when } & |\xi|>N .
\end{array}\right.
$$


The forms (8.3) have been established for $k=0,1,2$, by the theorems of the paper [9]. For the purpose we have in view it is important to observe that they are valid also for $k=3$. That can be done as follows. The functions $y_{h, j}$ are known $[9,(5.9)]$ to have the forms

$$
\begin{aligned}
\frac{d^{k} y_{h, 0}}{d z^{k}} & =\lambda^{2 k / 3} \zeta^{-2 \mu-2 k / 3} E(z, \lambda), \\
\frac{d^{k} y_{h, 1}}{d z^{k}} & =\lambda^{2 k / 8} \zeta^{\mu-1 / 2+k / 3} E(z, \lambda), \\
\frac{d^{k} y_{h, 2}}{d z^{k}} & =\lambda^{2 k / 3 \zeta^{\mu-1 / 2+k / 3} E(z, \lambda),} \quad k=0,1,2,3 .
\end{aligned}
$$

Inasmuch as these functions fulfill a differential equation of the form

$$
L(y)=\lambda^{-m_{1}-1}\left[E(z, \lambda) y^{\prime \prime}+\lambda E(z, \lambda) y^{\prime}+\lambda^{2} E(z, \lambda) y\right],
$$

it follows that

$$
\begin{aligned}
& L\left(y_{h, 0}\right)=\lambda^{-m_{1}+1} \zeta^{-2 \mu} E(z, \lambda), \\
& L\left(y_{h, 1}\right)=\lambda^{-m_{1}+1} \zeta^{\mu-1 / 2} e^{2 \zeta} E(z, \lambda), \\
& L\left(y_{h, 2}\right)=\lambda^{-m_{1}+1} \zeta^{\mu-1 / 2} e^{-2 \zeta} E(z, \lambda) .
\end{aligned}
$$

Therewith the final term of the formal identity

$$
u_{h, j}^{\prime \prime \prime}-y_{h, j}^{\prime \prime \prime}=-\lambda^{2} p\left[u_{h, j}^{\prime}-y_{h, j}^{\prime}\right]-\lambda^{2} q\left[u_{h, j}-y_{h, j}\right]-L\left(y_{h, j}\right),
$$

is appraised.

The appraisal of $\left[u_{h, j}-y_{h, j}\right]$ may be taken directly from the relations (8.3), and the same may be done for $\left[u_{h, j}^{\prime}-y_{h, j}^{\prime}\right]$. When this latter is coupled with the fact that $p_{0}(z)$ is of the order $z$ which is to say of the order of $(\xi / \lambda)^{2 / 8}$, the relation (8.13) is seen to yield the respective forms (8.3) with $k=3$.

We find it convenient to denote the function $u_{3}(z, \lambda)$ of $(7.1)$ alternatively by $u_{h, 8}(z, \lambda)$, whatever the integer $h$ may be. This is for the sake of uniformity in the notation. The functions $\eta_{h, j}$ with $j=0,1,2,3$, that correspond to the $u_{h, j}$ through the formulas (2.3) and (2.9), are then a complete set of solutions of the related equation. We have given their forms explicitly to the extent of all terms up to those of the $m$ th degree in $1 / \lambda$.

9. The enlistment of an integral equation. With the purpose of setting up an analytic procedure by which the unknown forms of the solutions of the given differential equation (1.1) may be inferred from the known solution forms of the related equation (6.3) we resort now to the device, which is familiar, of transforming the differential equation into an integral equation. We explain how this may be done in the following, giving first the formal motivation, and then, by a reversal of the reasoning, the actual method. For any solution $w(z)$ of the given differential equation (1.1) the function $l(w, z)$ 
given by the formula (6.4) is specific, and $w(z)$ is thus clearly a solution also of the nonhomogeneous differential equation

$$
\mathfrak{L}(W)+l(W, z)=l(w, z) .
$$

The reduced associate of this equation is the related equation (6.3). Therefore, $w(z)$ is expressible in terms of the solutions of the latter, in particular in terms of the functions $\eta_{h, j}(z)$, it being always assumed that $l(w, z)$ is known. The formula for this is obtainable by the method of variation of constants. With the Wronskian $W\left(\eta_{h, 0}, \eta_{h, 1}, \eta_{h, 2}, \eta_{h, 3} ; z\right)$ denoted in brief by $W(z)$, and with $\Delta_{j}(t)$ standing for the cofactor of the element $\eta_{h, j}(z)$ in the determinant $\Delta(z, t)$ given thus

$$
\Delta(z, t)=\left|\begin{array}{cccc}
\eta_{h, 0}(z) & \eta_{h, 1}(z) & \eta_{h, 2}(z) & \eta_{h, 3}(z) \\
\eta_{h, 0}(t) & \eta_{h, 1}(t) & \eta_{h, 2}(t) & \eta_{h, 3}(t) \\
\eta_{h, 0}^{\prime}(t) & \eta_{h, 1}^{\prime}(t) & \eta_{h, 2}^{\prime}(t) & \eta_{h, 3}^{\prime}(t) \\
\eta_{h, 0}^{\prime \prime}(t) & \eta_{h, 1}^{\prime \prime}(t) & \eta_{h, 2}^{\prime \prime}(t) & \eta_{h, 3}^{\prime \prime}(t)
\end{array}\right|
$$

this formula is

$$
w(z)=\sum_{j=0}^{3} c_{j} \eta_{h, j}(z)-\sum_{j=0}^{3} \eta_{h, j}(z) \int_{z_{j}}^{z} \frac{\Delta_{j}(t)}{W(t)} l(w, t) d t .
$$

The limits of integration $z_{j}$ are assignable as any appropriate values that are independent of $z$, and the constants $c_{j}$ are then specific of the solution $w(z)$ that is in question. The formula (9.2) is differentiable, and hence $l(w, z)$ itself can be formed from it. The result so obtained is the relation

$$
l(w, z)=\sum_{j=0}^{3} l\left(\eta_{h, j}, z\right)\left[c_{j}-\int_{z_{j}}^{z} \Omega_{j}(t) l(w, t) d t\right],
$$

with

$$
\Omega_{j}(t)=\frac{\Delta_{j}(t)}{W(t)} .
$$

This reasoning was merely heuristic, since it was built from the beginning on the assumption that $l(w, z)$ was known. A reversal of the reasoning yields, however, the following practicable method.

Let $f(z)$ be a solution of the integral equation

$$
f(z)=\sum_{j=0}^{3} l\left(\eta_{h, j}, z\right)\left[c_{j}-\int_{z_{j}}^{z} \Omega_{j}(t) f(t) d t\right],
$$

in which the constants $c_{j}$ and the limits $z_{j}$ have any assigned values. Then in terms of this function, and with the same $c_{j}$ and $z_{j}$, let the function $w(z)$ be given by the formula 


$$
w(z)=\sum_{j=0}^{3} \eta_{h, j}(z)\left[c_{j}-\int_{z_{j}}^{z} \Omega_{j}(t) f(t) d t\right] .
$$

We may see, as follows, that this function $w(z)$ is a solution of the given differential equation (1.1).

The sum

$$
\sum_{j=0}^{3} \eta_{h, j}(z) \Omega_{j}(t)
$$

has the value $\Delta(z, t) / W(t)$. The determinant $\Delta(z, t)$ and its first two partial derivatives with respect to $z$ vanish at $t=z$, and its third partial derivative has the value $-W(z)$ for that $t$. The derivatives of the formula (9.6) are accordingly

$$
\frac{d^{k} w}{d z^{k}}=\sum_{j=0}^{3} \frac{d^{k} \eta_{h, j}(z)}{d z^{k}}\left[c_{j}-\int_{z_{j}}^{z} \Omega_{j}(t) f(t) d t\right]+\delta_{k, 4} f(z),
$$

for $k=1,2,3,4$, with $\delta_{4,4}=1$ and $\delta_{k, 4}=0$, when $k \neq 4$. We obtain from this

$$
\mathscr{L}(w)=\sum_{j=0}^{3} \mathcal{L}\left(\eta_{h, j}\right)\left[c_{j}-\int_{z_{j}}^{z} \Omega_{j}(t) f(t) d t\right]+f(z) .
$$

By substituting for $f(z)$ its value, as that is taken from (9.5), the relation is given the form

$$
\mathscr{L}(w)=\sum_{j=0}^{3}\left[\mathcal{L}\left(\eta_{h, j}\right)+l\left(\eta_{h, j}, z\right)\right]\left[c_{j}-\int_{z_{j}}^{z} \Omega_{j}(t) f(t) d t\right],
$$

and in this each term on the right is zero, since each function $\eta_{h, j}$ fulfills the equation (6.3).

By the method that has thus been described, the analyses that lead to the forms of solutions of the differential equation (1.1), are effectively referred to considerations of the solutions of respective integral equations (9.5). This is the method by which we shall proceed below.

10. Some appraisals. For the analyses of the equations (9.5) and (9.6) with which we shall wish to deal, we must draw to hand the forms of the functions $\eta_{h, j}(z)$, and of their respective combinations $l\left(\eta_{h, j}, z\right)$ and $\Omega_{j}(t)$. These forms will, however, not be needed to any such degree of explicitness as has been given in $\S 8$. The characters of their dominant terms generally suffice.

It was observed in $\S 8$ that the functions $d^{k} y_{h, j} / d z^{k}$, with $j=0,1,2,3$, have the forms (8.12). By the relations (8.3) in which, it will be recalled, $m_{1} \geqq 3$, so that $m_{1} \geqq k$, the respective functions $d^{k} u_{h, j} / d z^{k}$ are shown to have those same forms. The demonstration is extended to the corresponding functions $d^{k} \eta_{h, j} / d z^{k}$ by the relation (2.3). An appeal to the evaluations

$$
\zeta^{ \pm \mu}=\zeta^{ \pm \mu_{0}} E(z, \lambda)
$$


which follow from (8.9), leads thus to the first three of the appraisals

$$
\begin{aligned}
\frac{d^{k} \eta_{h, 0}}{d z^{k}} & =\lambda^{2 k / 3} \zeta^{-2 \mu_{0}-2 k / 3} E(z, \lambda), \\
\frac{d^{k} \eta_{h, 1}}{d z^{k}} & =\lambda^{2 k / 3} \zeta^{\mu_{0}-1 / 2+k / 3} e^{2 \zeta} E(z, \lambda), \\
\frac{d^{k} \eta_{h, 2}}{d z^{k}} & =\lambda^{2 k / 3} \zeta^{\mu 0-1 / 2+k / 3} e^{-2 \zeta} E(z, \lambda), \\
\frac{d^{k} \eta_{h, 3}}{d z^{k}} & =E(z, \lambda) .
\end{aligned}
$$

The fourth one of these is observable directly from the relations (2.7) and (7.1). By virtue of the formula (6.5), we find accordingly that

$$
\begin{aligned}
& l\left(\eta_{h, 0}, z\right)=\lambda^{-m+7 / 3} \zeta^{-2 \mu_{0}-4 / 3} E(z, \lambda), \\
& l\left(\eta_{h, 1}, z\right)=\lambda^{-m+7 / 3} \zeta^{\mu+1 / 6} e^{25} E(z, \lambda), \\
& l\left(\eta_{h, 2}, z\right)=\lambda^{-m+7 / 3} \zeta^{\mu_{0}+1 / 6} e^{-25} E(z, \lambda) . \\
& l\left(\eta_{h, 3}, z\right)=\lambda^{-m+1} E(z, \lambda) .
\end{aligned}
$$

In the Wronskian $W\left(u_{h, 0}, u_{h, 1}, u_{h, 2}, u_{h, 3} ; z\right)$, each element $u_{h, j}^{\prime \prime \prime}$ is replaceable by the respective form $L\left(u_{h, j}\right)$, since that replacement merely augments one row of a determinant by multiples of the other rows. Then since $L\left(u_{h, j}\right)=0$ for $j=0,1,2$, whereas, by (7.6),

$$
L\left(u_{h, 3}\right)=\lambda^{2-\delta}\left[1+\frac{E(z, \lambda)}{\lambda^{m+1}}\right],
$$

with ${ }^{(3)} \delta=0$, we see that this Wronskian has the value

$$
\lambda^{2-\delta \sigma}\left(u_{h, 0}, u_{h, 1}, u_{h, 2} ; z\right)\left[1+\frac{E(z, \lambda)}{\lambda^{m+1}}\right] .
$$

The Wronskian that remains in this expression involves only solutions of the differential equation $L(u)=0$. Its value is known $[9,(5.11)]$ to be $-6 \lambda^{2}[1+E(\lambda) / \lambda]$. Inasmuch as $D_{5}(z, \lambda)$ of $\S 6$ is also of the form $[1+E(z, \lambda) / \lambda]$, we draw from the relation (6.6) the evaluation

$$
w(z)=-6 \lambda^{4-\delta}\left[1+\frac{E(z, \lambda)}{\lambda}\right] .
$$

(3) We write the formula (10.3), and some subsequent ones, with the inclusion of an exponent $\delta$. The purpose of this is to make the formulas adaptable to the considerations of Part III of the paper, where the differential equations at issue are of an irregular type. Throughout the present part of the discussion, namely throughout Part II, in which the differential equation is of the regular type, the value of $\delta$ is always zero. 
The formula (9.4) now yields

$$
\begin{aligned}
& \Omega_{0}(t)=\lambda^{\delta-2} \zeta^{2 \mu_{0}}(t) E(t, \lambda), \\
& \Omega_{1}(t)=\lambda^{\delta-2} \zeta^{\mu_{0}-1 / 2}(t) e^{-2 \zeta(t)} E(t, \lambda), \\
& \Omega_{2}(t)=\lambda^{\delta-2} \zeta^{\mu_{0}-1 / 2}(t) e^{2 \zeta(t)} E(t, \lambda), \\
& \Omega_{3}(t)=\lambda^{\delta-2} E(t, \lambda) .
\end{aligned}
$$

The symbol $\zeta(t)$ denotes, of course, that function of $t$ which $\zeta$, in (8.11), is of $z$.

11. Paths of integration. A locus in the region $R_{z}$ has been referred to [9, $\S 7]$ as a $\gamma$-curve, if its image in $R_{\xi}$ under the mapping relation (8.1), is a broken line, namely a continuous chain of straight segments of which both the number and the slopes are bounded, on which the real part of $\xi$ is monotonic. We shall adopt that terminology here. Also we shall apply the designation " $\delta$-curve," to any locus which in $R_{\xi}$ appears as a circular arc centered at the origin joined to a segment of a radial line. Finally we shall refer to any locus that issues from the origin, and which appears as straight in $R_{\xi}$, as an "outward radius."

Any region $R_{z}^{\Lambda}$, that is associated with a $\lambda$-region $\Lambda$, has certain properties which are important, and which we here recall $[9$, p. 104]. Its image region $R_{\xi}^{\mathbf{A}}$ is star-shaped with respect to its origin. Therefore every point of it can be reached from the origin along an outward radius. Near the origin, it contains only a single segment of any arc on which $|\xi|$ is constant. Because of this it is possible to find within the region a point $z_{*}$ distinct from the origin, from which every point of the region can be reached along a $\delta$-curve. Finally every such region contains two points $z_{r}$ and $z_{l}$ whose salient properties are the following: that every point of the region can (for any $\lambda$ in $\Lambda$ ) be reached from $z_{r}$ along a $\gamma$-curve on which the real part of $\xi$ monotonically decreases, while every point can (for any $\lambda$ in $\Lambda$ ) be reached from $z_{l}$ along a $\gamma$-curve on which the real part of $\xi$ monotonically increases.

When $z$ is restricted to a region $R_{z}^{\Lambda}$, and the points $z_{j}$ are taken in that region, the paths of integration in the equations (9.5) and (9.6) are inconsequential, since the integrands are analytic. We therefore have the option to choose these paths so as to facilitate appraisals of the integrals. The paths that are found to be convenient for this are the outward radii and the $\gamma$ and $\delta$-curves.

As has already been indicated in (9.5) and (9.6), we shall designate the variable of integration always by $t$. We shall suppose a point $z_{*}$ to have been chosen. Since this is fixed in $R_{z}^{\mathbf{\Lambda}}$, its image point $\xi\left(z_{*}\right)$ is a multiple of $\lambda$. We may therefore assume that the relation $\left|\xi\left(z_{*}\right)\right|>N$ maintains, since it is assured when $|\lambda|$ is sufficiently large. Therefore, by $(8.11), \zeta\left(z_{*}\right)=\xi\left(z_{*}\right)$.

12. Some lemmas. For use in the analyses of the equations (9.5) and (9.6), it is convenient to have certain facts formulated in the manner of lemmas. 
Lemma 1. For any constant $\alpha$ whose real part is not negative, for $z_{*}$ in a region $R_{z}^{\Lambda}$, and for $t$ on the $\delta$-curve from $z_{*}$ to $z$, the function

$$
\zeta^{\alpha}(z) \zeta^{-\alpha}(t)
$$

is bounded uniformly, for $\lambda$ and $z$ in the associated regions $\Lambda, R_{z}^{\Lambda}$.

When $z$ is in that part of $R_{z}^{\Lambda}$ in which $|\xi| \leqq\left|\xi\left(z_{*}\right)\right|$, the $\delta$-curve is one along which $|\zeta(t)|$ is nonincreasing. Its smallest value is therefore $|\zeta(z)|$. Thus $\zeta(z) \zeta^{-1}(t)$ is in absolute value at most 1 . The same is true of its $\alpha$ th power (12.1). When $z$ is in the part of the region in which $|\xi|>\left|\xi\left(z_{*}\right)\right|$, the $\delta$-curve is one along which $|\zeta(t)|$ has its smallest value at $z_{*}$. Thus, since $\zeta(t)=\xi(t)$ along the path, we have

$$
\left|\zeta(z) \zeta^{-1}(t)\right| \leqq\left|\xi(z) \xi^{-1}\left(z_{*}\right)\right| \text {. }
$$

In this the right hand member is bounded, since it is independent of $\lambda$ and is analytic in the closed region $R_{\mathbf{z}}$.

Lemma 2. For any constant $\alpha$ whose real part is not positive, and for $t$ on the outward radius to $z$, the function (12.1) is uniformly bounded in the regions $\Lambda, R_{\mathbf{2}}^{\Lambda}$.

This follows directly from the fact that $|\zeta(t)| \leqq|\zeta(z)|$ along the path.

Lemma 3. For any complex constant $\alpha$ the function

$$
\zeta^{\alpha}(z) \zeta^{-\alpha}(t) e^{2 \zeta(z)-2 \zeta(t)},
$$

is uniformly bounded for $t$ on the $\gamma$-curve from $z_{r}$ to $z$, and the function

$$
\zeta^{\alpha}(z) \zeta^{\alpha}(t) e^{-2 \zeta(z)+2 \zeta(t)},
$$

is uniformly bounded for $t$ on the $\gamma$-curve from $z_{l}$ to $z$. This is a known lemma $[9, \S 8]$.

With the use of the symbol $E(z, t, \lambda)$ to signify generically a function that is bounded when $|\lambda|$ is sufficiently large, and that is analytic in $z$ and in $t$ for the domains of these variables that may be concerned, we have also

Lemma 4. An integral of the form

$$
\int\left|\lambda^{1 / 3} \zeta^{-1 / 3}(t) E(z, t, \lambda) d t\right|,
$$

in which the path of integration is an outward radius, $a \delta$-curve, or a $\gamma$-curve, is uniformly bounded for $\lambda$ and $z$ in the associated regions $\Lambda, R_{z}^{\Lambda}$.

From the formulas (8.1) and (8.7) it is readily found that

$$
\frac{d \xi}{d z}=\frac{(i \lambda)^{2 / 3}(3 \xi)^{1 / 3}}{2 \Psi(z)}
$$


From this, and the fact that $\Psi(z)$ is bounded, it follows that $|d t|$ is less than a certain fixed multiple of $|d \xi(t)| /\left|\lambda^{2 / 8} \xi^{1 / 3}(t)\right|$. Since also $|\zeta| \geqq|\xi|$, the integral (12.4) is seen to be less than some specific multiple of the integral

$$
\frac{1}{|\lambda|^{1 / 3}} \int^{z} \frac{|d \xi|}{|\xi|^{2 / 3}}
$$

taken over the same path. Now on an outward radius, or on the straight segment of a $\delta$-curve, $|d \xi|$ and $d|\xi|$ are equal, except possibly for sign, and the integration accordingly contributes to the total value of (12.6) the difference of two amounts that are of the form $|\xi / \lambda|^{1 / 3}$. This contribution is independent of $\lambda$ and is bounded in $R_{\mathbf{z}}$. On the circular arc of a $\delta$-curve we have $|d \xi|$ $=|\xi| d \theta$, and hence the integration contributes to the total value of (12.6) an amount that is less than $2 \pi|\xi / \lambda|^{1 / 3}$. Consider, finally, the integration over any segment of a $\gamma$-curve. On the straight line along which this segment lies, let $\xi_{0}$ be the point nearest the origin. Then $|\xi| \geqq\left|\xi-\xi_{0}\right|$, whereas $|d \xi|$ $= \pm d\left|\xi-\xi_{0}\right|$. The contribution to the value (12.6) is thus seen to be no more than a difference of two certain values $\left|\left(\xi-\xi_{0}\right) / \lambda\right|^{1 / 3}$. Since the number of such segments is bounded, the lemma is proved.

13. A solution $w_{h, 0}(z)$. We turn now to the intended applications of the integral equation method that was set up in \$9. Beginning with the integral equation (9.5), we shall, in this section and in $\$ \$ 14$ and 15 , make specific choices of the integration limits $z_{j}$ and of the constants $c_{j}$. Corresponding to these, the equation has, in each instance, a solution $f(z)$. The functional form of this we shall appraise. With the function $f(z)$ at hand we shall then turn to the formula (9.6), which yields a respective function $w(z)$. This was shown in $\S 9$ to be a solution of the given differential equation. The form of this solution, and of its derivatives, since the formula (9.6) is differentiable, will thus be brought to hand.

In the integral equation (9.5), let the limits of integration be assigned thus

$$
z_{1}=z_{r}, \quad z_{2}=z_{l},
$$

$$
z_{0}=z_{3}=\left\{\begin{array}{l}
z_{*}, \text { if the real part of }\left[\mu_{0}+1 / 2\right] \text { is positive, } \\
0, \text { if the real part of }\left[\mu_{0}+1 / 2\right] \text { is not positive. }
\end{array}\right.
$$

Therewith let the choice of constants $c_{0}=1, c_{j}=0$, for $j \neq 0$, be made. With $f_{0}$ written in the place of $f$, the equation is then

$$
f_{0}(z)=l\left(\eta_{h, 0}, z\right)-\sum_{j=0}^{3} l\left(\eta_{h, j}, z\right) \int_{z_{j}}^{z} \Omega_{j}(t) f_{0}(t) d t .
$$

We shall consider this equation for $\lambda$ and $z$ in any pair of associated regions $\Lambda, R_{z}^{\Lambda}$, the integer $h$ being the one that is attached to them by the relations (8.2). The points $z_{r}, z_{l}$ and $z_{*}$ shall be those that were referred to as such in \$11. The paths of integration from $z_{r}$ and $z_{l}$ shall be $\gamma$-curves, that from $z_{*}$ 
a $\delta$-curve, and that from the origin an outward radius.

We begin by multiplying the equation (13.2) through by $\lambda^{m-7 / 3} \zeta^{2 \mu_{0}+4 / 8}$. It can then be expressed in terms of the functions $Y_{j}(z)$ and $F_{0}(z)$ that are given by the formulas

$$
\begin{array}{ll}
Y_{0}(z)=\lambda^{m-7 / 3} \zeta^{2 \mu_{0}+4 / 3} l\left(\eta_{h, 0}, z\right), & Y_{2}(z)=\lambda^{m-7 / 3} \zeta^{\mu_{0}-1 / 6} e^{2 \zeta l\left(\eta_{h, 2}, z\right),} \\
Y_{1}(z)=\lambda^{m-7 / 3} \zeta^{-\mu_{0}-1 / 6} e^{-2 \zeta} l\left(\eta_{h, 1}, z\right), & Y_{3}(z)=\lambda^{m-1} l\left(\eta_{h, 3}, z\right),
\end{array}
$$

and

$$
F_{0}(z)=\lambda^{m-7 / 3} \zeta^{2 \mu_{0}+4 / 3} f_{0}(z)
$$

The form that is thus given it is

$$
F_{0}(z)=Y_{0}(z)+\frac{1}{\lambda^{m-\delta}} \int^{z} K_{0}(z, t, \lambda) F_{0}(t) d t,
$$

the integration still extending over the several paths that are more explicitly indicated in (13.2). The formulas for the kernel $K_{0}(z, t, \lambda)$ on these several paths are the following ones

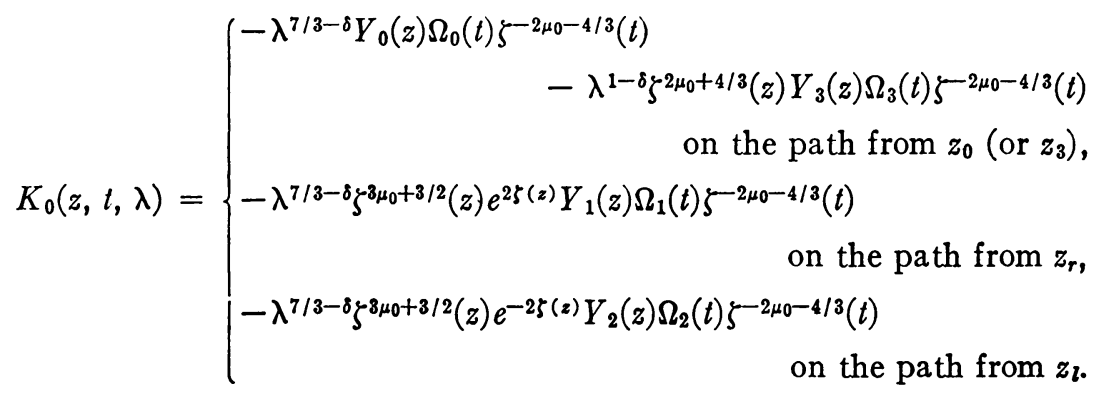

The functions (13.3) are all bounded, namely are all of the type $E(z, \lambda)$. This is shown by the evaluations (10.2). By the use of the relations (10.5) it can be shown, therefore, that $K_{0}(z, t, \lambda)$ has the forms

(13.7) $K_{0}(z, t, \lambda)=\left\{\begin{array}{r}\left.\text { on the path from } z_{0} \text { (or } z_{3}\right), \\ \lambda^{1 / 3} \zeta^{-1 / 3}(t)\left\{\zeta^{3 \mu_{0}+3 / 2}(z) \zeta^{-3 \mu_{0}-3 / 2}(t) e^{2 \zeta(z)-2 \zeta(t)}\right\} E(z, t, \lambda) \\ \text { on the path from } z_{r}, \\ \lambda^{1 / 3} \zeta^{-1 / 3}(t)\left\{\zeta^{3 \mu_{0}+3 / 2}(z) \zeta^{-3 \mu_{0}-3 / 2}(t) e^{-2 \zeta(z)+2 \zeta(t)}\right\} E(z, t, \lambda) \\ \text { on the path from } z_{l} .\end{array}\right.$ 
In these evaluations each quantity within braces is bounded on the path that is in question. Where that is not obvious, it is assured by the lemmas of $\$ 12$. It follows, therefore, by the Lemma 4 , that there exists a constant $M_{0}$ with which the relation

$$
\int^{z}\left|K_{0}(z, t, \lambda) d t\right|<M_{0}
$$

maintains. On the basis of this, familiar reasoning $[9, \S 9]$ may be applied to establish the fact that the equation (13.5) has, when $m>\delta$, a solution $F_{0}(z)$ that is bounded, namely that is of the type $E(z, \lambda)$. The relation (13.4) shows accordingly that

$$
f_{0}(z)=\lambda^{-m+7 / 3 \zeta^{-2 \mu_{0}-4 / 3} E(z, \lambda) .}
$$

We turn now to the equation (9.6). With the functions $\theta_{0, j}(z)$ given by the formulas

$$
\theta_{0, j}(z)=\int_{z j}^{z} \Omega_{j}(t) f_{0}(t) d t, \quad j=0,1,2,3,
$$

that equation defines the function $w_{h, 0}(z)$, and its first three derivatives, thus

$$
\frac{d^{k} w_{h, 0}}{d z^{k}}=\frac{d^{k} \eta_{h, 0}}{d z^{k}}-\sum_{j=0}^{3} \theta_{0, j}(z) \frac{d^{k} \eta_{h, j}}{d z^{k}}, \quad k=0,1,2,3 .
$$

As to the functions (13.10), we may use the appraisals (10.5) and (13.9) to show that they have the forms

$$
\begin{aligned}
\theta_{0,0}(z)= & \lambda^{-m+\delta} \int_{z_{0}}^{z} \lambda^{1 / 3} \zeta^{-1 / 3}(t)\left\{\frac{E(t, \lambda)}{\zeta(t)}\right\} d t, \\
\theta_{0,1}(z)= & \lambda^{-m+\delta} \zeta^{3 \mu_{0}-3 / 2}(z) e^{-2 \zeta(z)} \\
& \cdot \int_{z_{r}}^{z} \lambda^{1 / 3} \zeta^{-1 / 3}(t)\left\{\zeta^{3 \mu_{0}+3 / 2}(z) \zeta^{-3 \mu_{0}-3 / 2}(t) e^{2 \zeta(z)-2 \zeta(t)}\right\} E(t, \lambda) d t, \\
\theta_{0,2}(z)= & \lambda^{-m+\delta} \zeta^{3 \mu_{0}-3 / 2}(z) e^{2 \zeta(z)} \\
& \cdot \int_{z_{1}}^{s} \lambda^{1 / 3} \zeta^{1 / 3}(t)\left\{\zeta^{3 \mu_{0}+3 / 2}(z) \zeta^{-3 \mu_{0}-3 / 2}(t) e^{-2 \zeta(z)+2 \zeta(t)}\right\} E(t, \lambda) d t, \\
\theta_{0,3}(z)= & \lambda^{-m+8} \zeta^{2 \mu_{0}-1}(z) \int_{z_{3}}^{s} \lambda^{1 / 3} \zeta^{-1 / 3}(t)\left\{\zeta^{2 \mu_{0}+1}(z) \zeta^{-2 \mu_{0}-1}(t)\right\} E(t, \lambda) d t .
\end{aligned}
$$

The lemmas of $\$ 12$ are applicable to these, to show first that all quantities that appear within braces are bounded on the respective integration paths, and then that the structures of the functions $\theta_{0, j}(z)$ are, in fact, the following 


$$
\begin{aligned}
& \theta_{0,0}(z)=\lambda^{-m+\delta} E(z, \lambda), \\
& \theta_{0,1}(z)=\lambda^{-m+\delta} \zeta^{3 \mu_{0}-3 / 2} e^{-2 \zeta} E(z, \lambda), \\
& \theta_{0,2}(z)=\lambda^{-m+\delta} \zeta^{3 \mu_{0}-3 / 2} e^{2 \zeta} E(z, \lambda), \\
& \theta_{0,3}(z)=\lambda^{-m+\delta} \zeta^{2 \mu_{0}-1} E(z, \lambda) .
\end{aligned}
$$

These evaluations, along with those of (10.1), may be substituted into the formulas (13.11). The conclusion that is thereby reached is the following one.

THEOREM 1. For $\lambda$ and $z$ in any pair of associated regions, the differential equation (1.1) has a solution $w_{h, 0}(z)$ to which the formulas

$$
\frac{d^{k} w_{h, 0}}{d z^{k}}=\frac{d^{k} \eta_{h, 0}}{d z^{k}}+\frac{5^{2 \mu 0-2 k / 3} E(z, \lambda)}{\lambda^{m-\delta-2 k / 3}}, \quad k=0,1,2,3,
$$

apply.

The significance of the symbols in these formulas is recalled to be the following:

For $\lambda$ and $z$ in associated regions (defined in $[9, \S 7]$ ) the relations (8.2) are fulfilled by some integer $h$. Thereby $h$ is identified.

The function $\eta_{h, 0}$ is given by (2.3). Its coefficients $Q(z, \lambda)$ and $\mathcal{C}(z, \lambda)$ are determined in $\S \S 3,4$ and 5 , and the function $u_{h, 0}(z)$ is described in $\S 8$.

The meaning of $\zeta$ is given by (8.11) and (8.1).

The value of $\mu_{0}$ is given by (8.9) and (1.9).

If the differential equation (1.1) is regular, namely if $\rho$, as given in (1.9), is not zero or a positive integer, the value of $\delta$ is zero.

The symbol $E(z, \lambda)$ signifies a function that is analytic in $z$ and uniformly bounded when $|\lambda|$ is sufficiently large.

The integer $m$ can be arbitrarily assigned.

14. A pair of solutions $w_{h, 1}(z), w_{h, 2}(z)$. When each integration limit is assigned to be $z_{l}$, and the choice of constants $c_{1}=1, c_{j}=0$, for $j \neq 1$, is therewith made, the integral equation (9.5) is specifically

$$
f_{1}(z)=l\left(\eta_{h, 1}, z\right)-\sum_{j=0}^{3} l\left(\eta_{h, j}, z\right) \int_{z_{l}}^{z} \Omega_{j}(t) f_{1}(t) d t .
$$

The processes and reasoning we shall apply to this are analogous to those of \$13. The equation, after being multiplied by $\lambda^{m-7 / 3} \zeta^{-\mu_{0}-1 / 6} e^{-25}$ appears as

$$
F_{1}(z)=Y_{1}(z)+\frac{1}{\lambda^{m-\delta}} \int_{z l}^{z} K_{1}(z, t, \lambda) F_{1}(t) d t
$$

with

$$
F_{1}(z)=\lambda^{m-7 / 3 \zeta} \zeta^{\mu_{0}-1 / 6} e^{-2 \zeta} f_{1}(z),
$$

and with the kernel 


$$
\begin{aligned}
K_{1}(z, t, \lambda)= & -\lambda^{7 / 3-\delta} \zeta^{-3 \mu_{0}-3 / 2}(z) e^{-2 \zeta(z)} Y_{0}(z) \Omega_{0}(t) \zeta^{\mu 0+1 / 6}(t) e^{2 \zeta(t)} \\
& -\lambda^{7 / 3-\delta} Y_{1}(z) \Omega_{1}(t) \zeta^{\mu_{0}+1 / 6}(t) e^{2 \zeta(t)} \\
& -\lambda^{7 / 3-\delta} e^{-4 \zeta(z)} Y_{2}(z) \Omega_{2}(t) \zeta^{\mu_{0}+1 / 6}(t) e^{2 \zeta(t)} \\
& -\lambda^{1-\delta} \zeta^{\mu-1 / 16}(z) e^{-2 \zeta(z)} Y_{3}(z) \Omega_{3}(t) \zeta^{\mu_{0}+1 / 6}(t) e^{2 \zeta(t)} .
\end{aligned}
$$

The evaluations (10.5), and the boundedness of the functions $Y_{j}(z)$, show this to be of the form

$$
\begin{aligned}
K_{1}(z, t, \lambda)= & \lambda^{1 / 3} \zeta^{-1 / 3}(t)\left\{\zeta^{-3 \mu_{0}-3 / 2}(z) \zeta^{3 \mu_{0}+3 / 2}(t) e^{-2 \zeta(z)+2 \zeta(t)}\right\} \frac{E(z, t, \lambda)}{\zeta(t)} \\
& +\lambda^{1 / 3} \zeta^{-1 / 3}(t) E(z, t, \lambda)+\lambda^{1 / 3} \zeta^{-1 / 3}(t)\left\{e^{-4 \zeta(z)+4 \zeta(t)}\right\} E(z, t, \lambda) \\
& +\lambda^{-1}\left\{\zeta^{-\mu_{0}+1 / 6}(z) \zeta^{\mu_{0}+1 / 6}(t) e^{-2 \zeta(z)+2 \zeta(t)}\right\} E(z, t, \lambda) .
\end{aligned}
$$

The lemmas of $\$ 12$ show that the quantities within braces are bounded, and then further that there exists a constant $M_{1}$ such that

$$
\int_{z l}^{z}\left|K_{1}(z, t, \lambda) d t\right|<M_{1} \text {. }
$$

There is therefore a bounded solution $F_{1}(z)$ of the equation (14.2), and thus, by (14.3),

$$
f_{1}(z)=\lambda^{-m+7 / 3 \zeta^{\mu}+1 / 6} e^{2 \zeta} E(z, \lambda) .
$$

In terms of the functions

$$
\theta_{1, j}(z)=\int_{z_{\imath}}^{z} \Omega_{j}(t) f_{1}(t) d t, \quad j=0,1,2,3,
$$

the equation (9.6) now defines $w_{h, 1}(z)$, and gives its derivatives, by the formulas

$$
\frac{d^{k} w_{h, 1}}{d z^{k}}=\frac{d^{k} \eta_{h, 1}}{d z^{k}}-\sum_{j=0}^{8} \theta_{1, j}(z) \frac{d^{k} \eta_{h, j}}{d z^{k}}, \quad k=0,1,2,3 .
$$

The evaluations (10.5) and (14.5) show that the forms of the functions $\theta_{1, j}(z)$ are

$$
\begin{aligned}
\theta_{1,0}(z)= & \lambda^{-m+\delta} \zeta^{3 \mu_{0}+1 / 2} e^{2 \zeta} \\
& \int_{z l}^{z} \lambda^{1 / 3} \zeta^{-1 / 3}(t)\left\{\zeta^{-3 \mu_{0}-1 / 2}(z) \zeta^{3 \mu_{0}+1 / 2}(t) e^{-2 \zeta(z)+2 \zeta(t)}\right\} E(z, t, \lambda) d t, \\
\theta_{1,1}(z)= & \lambda^{-m+\delta} \int_{z_{l}}^{z} \lambda^{1 / 3} \zeta^{-1 / 3}(t) E(z, t, \lambda) d t, \\
\theta_{1,2}(z)= & \lambda^{-m+\delta} e^{4 \zeta} \int_{z l}^{z} \lambda^{1 / 3 \zeta} \zeta^{-1 / 3}(t)\left\{e^{-4 \zeta(z)+4 \zeta(t)}\right\} E(z, t, \lambda) d t,
\end{aligned}
$$




$$
\begin{aligned}
\theta_{1,3}(z)= & \lambda^{-m+\delta} \zeta^{\mu_{0}+1 / 2} e^{2 \zeta} \\
& \cdot \int_{z_{l}}^{z} \lambda^{1 / 3} \zeta^{-1 / 3}(t)\left\{\zeta^{\mu_{\sigma}-1 / 2}(z) \zeta^{\mu_{0}+1 / 2}(t) e^{-2 \zeta(z)+2 \zeta(t)}\right\} E(z, t, \lambda) d t .
\end{aligned}
$$

The lemmas of $\$ 12$ show that each integral here involved is of the type $E(z, \lambda)$. Therefore we may draw from the relations (14.6) the following conclusion:

THEOREM 2. For $\lambda$ and $z$ in any pair of associated regions, the differential equation (1.1) has a solution $w_{h, 1}(z)$ to which the formulas

$$
\frac{d^{k} w_{h, 1}}{d z^{k}}=\frac{d^{k} \eta_{h, 1}}{d z^{k}}+\frac{\zeta^{\mu_{0}-1 / 2+k / 3} e^{2 \zeta} E(z, \lambda)}{\lambda^{m-\delta-2 k / 3}}, \quad k=0,1,2,3,
$$

apply.

The significance of the symbols was recalled in connection with Theorem 1.

The integral equation (9.5), with each integration limit assigned to be $z_{r}$, and with the constants $c_{2}=1, c_{j}=0$, for $j \neq 2$, is

$$
f_{2}(z)=l\left(\eta_{h, 2}, z\right)-\sum_{j=0}^{3} l\left(\eta_{h, j}, z\right) \int_{z_{r}}^{z} \Omega_{j}(t) f_{2}(t) d t .
$$

The discussion given above of the equation (14.1), is essentially adaptable to the equation (14.8) by making the replacements of $z_{l}$ by $z_{r}$, and of $e^{\xi}$ by $e^{-r}$. The conclusion that is obtainable in that way is the following one.

TheOREM 3. For $\lambda$ and $z$ in any pair of associated regions, the differentia equation (1.1) has a solution $w_{h, 2}(z)$ to which the formulas

$$
\frac{d^{k} w_{h, 2}}{d z^{k}}=\frac{d^{k} \eta_{h, 2}}{d z^{k}}+\frac{\zeta^{\mu-1 / 2+k / 3} e^{-2 \zeta} E(z, \lambda)}{\lambda^{m-\delta-2 k / 3}}, \quad k=0,1,2,3,
$$

apply.

The significance of the symbols was recalled in connection with Theorem 1.

15. A solution $w_{h, 3}(z)$. A final form in which we shall consider the integral equation (9.5) is

$$
f_{3}(z)=l\left(\eta_{h, 3}, z\right)-\sum_{j=0}^{8} l\left(\eta_{h, j}, z\right) \int_{a_{j}}^{z} \Omega_{j}(t) f_{3}(t) d t,
$$

in which the integration limits are assigned thus 


$$
z_{1}=z_{r}, \quad z_{2}=z_{l},
$$

$$
z_{0}=z_{3}= \begin{cases}z_{*}, & \text { if the real part of }\left[\mu_{0}+2 / 3\right] \text { is negative, } \\ 0, & \text { if the real part of }\left[\mu_{0}+2 / 3\right] \text { is not negative. }\end{cases}
$$

After multiplication by $\lambda^{m-1}$ the equation appears as

$$
F_{3}(z)=Y_{3}(z)+\frac{1}{\lambda^{m-\delta}} \int^{z} K_{3}(z, t, \lambda) F_{3}(t) d t,
$$

with

$$
F_{3}(z)=\lambda^{m-1} f_{3}(z),
$$

and with the kernel

$$
K_{3}(z, t, \lambda)=\left\{\begin{array}{rr}
-\lambda^{7 / 3-\delta} \zeta^{2 \mu_{0}-4 / 3}(z) Y_{0}(z) \Omega_{0}(t)-\lambda^{1-\delta} Y_{3}(z) \Omega_{3}(t), \\
\quad \text { on the path from } z_{0}\left(\text { or } z_{3}\right), \\
-\lambda^{7 / 3-\delta} \zeta^{\mu_{0}+1 / 6}(z) e^{2 \zeta(z)} Y_{1}(z) \Omega_{1}(t), & \text { on the path from } z_{r}, \\
-\lambda^{7 / 3-\delta} \zeta^{\mu_{0}+1 / 6}(z) e^{-2 \zeta(z)} Y_{2}(z) \Omega_{2}(t), & \text { on the path from } z_{l} .
\end{array}\right.
$$

The form of this kernel is

$$
K_{3}(z, t, \lambda)=\left\{\begin{array}{cc}
\lambda^{1 / 3} \zeta^{-1 / 3}(t)\left\{\zeta^{-2 \mu_{0}-4 / 3}(z) \zeta^{2 \mu_{0}+4 / 3}(t)\right\} \frac{E(z, t, \lambda)}{\zeta(t)}+\frac{E(z, t, \lambda)}{\lambda}, \\
\text { on the path from } z_{0} \\
\lambda^{1 / 3} \zeta^{-1 / 3}(t)\left\{\zeta^{\mu_{0}+1 / 6}(z) \zeta^{-\mu_{0}-1 / 6}(t) e^{2 \zeta(z)-2 \zeta(t)}\right\} & E(z, t, \lambda), \\
& \text { on the path from } z_{r}, \\
\lambda^{1 / 3} \zeta^{-1 / 3}(t)\left\{\zeta^{\mu_{0}+1 / 6}(z) \zeta^{\mu_{0}-1 / 6}(t) e^{-2 \zeta(z)+2 \zeta(t)}\right\} & E(z, t, \lambda), \\
& \text { on the path from } z_{l} .
\end{array}\right.
$$

The reasoning that may be applied to this, to show that $F_{3}(z)$ is bounded, proceeds as in $\$ 13$. The conclusion is that

$$
f_{3}(z)=\lambda^{-m+1} E(z, \lambda) \text {. }
$$

The formula (9.6) now takes the form

$$
\frac{d^{k} w_{h, 3}}{d z^{k}}=\frac{d^{k} \eta_{h, 3}}{d z^{k}}-\sum_{j=0}^{8} \theta_{3, j}(z) \frac{d^{k} \eta_{h, j}}{d z^{k}}, \quad k=0,1,2,3,
$$

with the coefficients

$$
\theta_{3, j}(z)=\int_{z j}^{z} \Omega_{j}(t) f_{3}(t) d t, \quad j=0,1,2,3 .
$$

We find that these latter are of the forms 


$$
\begin{aligned}
& \theta_{3,0}(z)=\lambda^{-m-4 / 3+\delta} \zeta^{2 \mu_{0}+4 / 3} \int_{z_{0}}^{z} \lambda^{1 / 3} \zeta^{-1 / 3}(t)\left\{\zeta^{-2 \mu_{0}-4 / 3}(z) \zeta^{2 \mu_{0}+4 / 3}(t)\right\} \frac{E(z, t, \lambda)}{\zeta(t)} d t, \\
& \theta_{3,1}(z)=\lambda^{-m-4 / 3+\delta} \zeta^{-\mu_{0}-1 / 6} e^{-2 \zeta} \\
& \cdot \int_{z_{r}}^{z} \lambda^{1 / 3} \zeta^{-1 / 3}(t)\left\{\zeta^{\mu_{0}+1 / 6}(z) \zeta^{\mu_{0}-1 / 6}(t) e^{2 \zeta(z)-2 \zeta(t)}\right\} E(z, t, \lambda) d t, \\
& \theta_{3,2}(z)=\lambda^{-m-4 / 3+\delta} \zeta^{-\mu 0-1 / 6} e^{2 \zeta} \\
& \cdot \int_{z_{l}}^{z} \lambda^{1 / 3} \zeta^{-1 / 3}(t)\left\{\zeta^{\mu_{0}+1 / 6}(z) \zeta^{\mu_{0}-1 / 6}(t) e^{-2 \zeta(z)+2 \zeta(t)}\right\} E(z, t, \lambda) d t, \\
& \theta_{3,3}(z)=\lambda^{-m-1+\delta} \int_{z_{3}}^{z} E(z, t, \lambda) d t .
\end{aligned}
$$

In these forms each integral is a function of the type $E(z, \lambda)$. The equations (15.7) therefore permit the conclusion:

TheOREM 4. For $\lambda$ and $z$ in any pair of associated regions, the differential equation (1.1) has a solution $w_{h, 3}(z)$ to which the formulas

$$
\begin{aligned}
& \frac{d^{k} w_{h, 3}}{d z^{k}}=\frac{d^{k} \eta_{h, 3}}{d z^{k}}+\frac{\zeta^{4 / 3-2 k / 3} E(z, \lambda)+\zeta^{-2 / 3+k / 3} E(z, \lambda)}{\lambda^{m-4 / 3-2 k / 3-\delta}}+\frac{E(z, \lambda)}{\lambda^{m+1-\delta}}, k=0,1,2,3, \\
& \text { apply. }
\end{aligned}
$$

The significance of the symbols was recalled in connection with Theorem 1.

\section{Part III. Differential equations of IRREgular type- AN EQUATION OF HYDRODYNAMICS}

16. Some formal relations. The analysis given in Part II above was restricted to differential equations of the type that was defined to be regular, namely to equations (1.1) for which the value of the constant $\rho$, given by the formula (1.9), is neither zero nor a positive integer. It has therefore left for consideration in this part of the paper the equations of an irregular type. These constitute a large and important class of differential equations (1.1). That will be recognized at once from the fact that they include, among others, all those equations that involve no term in the first derivative. The hydrodynamical equation (1.4) is irregular by virtue of that feature.

For a differential equation (1.1) of the regular type, the limiting form as $\lambda \rightarrow \infty$, namely the equation

$$
P_{0}(z) w^{\prime \prime}+Q_{0}(z) w^{\prime}+R_{0}(z) w=0,
$$

necessarily has a singular point at the origin. In the instance of an equation of the irregular type, that may or may not be the case; the origin may be 
either an ordinary point or a singular point, and in the latter event the singularity may be actual or only apparent. In other terms, for an irregular differential equation the limiting form (16.1) may admit just one analytic solution, or it may admit only such solutions. These alternatives, although they are not the whole criteria, will be seen to supply the initial earmarks for a certain categorical classification of irregular differential equations. For the definitive classification the coefficients of the equation (1.1) aside from those that appear in (1.6) will also be significant.

In terms of $y$, where $y=w / \alpha_{0}(z)$, the differential equation (16.1) appears as

$$
P_{0} y^{\prime \prime}+\left[Q_{0}+2 P_{0} \frac{\alpha_{0}^{\prime}}{\alpha_{0}}\right] y^{\prime}+\left[P_{0} \frac{\alpha_{0}^{\prime \prime}}{\alpha_{0}}+Q_{0} \frac{\alpha_{0}^{\prime}}{\alpha_{0}}+R_{0}\right] y=0 .
$$

This, however, is actually the equation

$$
p_{0} y^{\prime \prime}+\left[p_{0}^{\prime}+q_{0}\right] y^{\prime}+q_{0}^{\prime} y=0,
$$

as may be verified by substituting for $R_{0}$ the value given it by the equation (3.3), and then heeding the relations (3.2) and (3.5). The equation (16.2), however, is integrable, since its left-hand member is a perfect derivative. A first integral of it is the equation

$$
p_{0} y^{\prime}+q_{0} y=c,
$$

and with $c=0$, and $c=1$, respectively, this admits the solutions $\varepsilon_{0}(z)$ and $G_{0}(z)$, where

$$
\begin{aligned}
\varepsilon_{0}(z) & =\exp \left(-\int \frac{q_{0}(z)}{p_{0}(z)} d z\right), \\
G_{0}(z) & =\varepsilon_{0}(z) \int \frac{d z}{p_{0}(z) \varepsilon_{0}(z)} .
\end{aligned}
$$

These functions are therefore solutions of the equation (16.2), and their products by $\alpha_{0}(z)$ are solutions of the differential equation (16.1).

The function $\varepsilon_{0}(z)$ is in every instance analytic. It has a zero of the order $\rho$ at the origin, since $-q_{0}(0) / p_{0}^{\prime}(0)=\rho$. The function $G_{0}(z)$ is in some instances analytic and in other instances not so. When the latter is the case, it is because $G_{0}(z)$ includes a constant multiple of the product $\varepsilon_{0}(z) \log z$, which is singular at $z=0$.

The use of the form (7.1) for a function $u_{3}(z, \lambda)$ led, in $\$ 7$, to the equation (7.4), and this latter equation was assured of an analytic solution, because it was there assumed that $\rho$ was not zero or a positive integer. Our assumption now is to the contrary, since we are here considering irregular differential equations (1.1), and because of that the deductions of $\$ 7$ are not invariably applicable. A modification of them must be resorted to, and to that end we begin by taking for $u_{3}(z, \lambda)$ a new formula, namely 


$$
u_{3}(z, \lambda)=\sum_{n=0}^{m+\delta} \frac{U_{n}(z)}{\lambda^{n}}
$$

in which $\delta$ is a non-negative integer for which a determination will be given below. The substitution of this form into the expression $L(u)$ yields the result

$$
L\left(u_{3}\right)=\lambda^{2} \sum_{n=0}^{2 m^{\prime \prime}} \frac{p_{0} U_{n}^{\prime}+q_{0} U_{n}+H_{n}(U)}{\lambda^{n}},
$$

in which $2 m^{\prime \prime}$ is the larger one of the integers $2(m+\delta)$ and $(m+\delta)+2$, and

$$
H_{n}(U) \equiv U_{n-2}^{\prime \prime \prime}+\sum_{j=1}^{n}\left[p_{j} U_{n-j}^{\prime}+q_{j} U_{n-j}\right]
$$

It is to be understood, of course, that any symbol $p_{j}$ or $q_{j}$ whose subscript is not of the set $0,1,2, \cdots, m$, and any symbol $U_{j}$ whose subscript is not of the set $0,1,2, \cdots,(m+\delta)$, is to be assigned the value zero. The condition that the first $(m+\delta+1)$ terms of the sum (16.5) have respective constant values $k_{n}$ is, therefore, that the functions $U_{n}(z)$ of (16.4) fulfill in turn the equations

$$
p_{0} U_{n}^{\prime}+q_{0} U_{n}=k_{n}-H_{n}(U) \quad n=0,1,2, \cdots,(m+\delta) .
$$

These differential equations have the general solutions

$$
U_{n}(z)=\varepsilon_{0}(z) \int \frac{k_{n}-H_{n}(U)}{p_{0} \varepsilon_{0}} d z+c_{n} \varepsilon_{0}(z),
$$

the $c_{n}$ being arbitrary constants of integration. We shall show that in terms of the function sets

$$
\begin{aligned}
& \varepsilon_{0}(z), \varepsilon_{1}(z), \cdots, \varepsilon_{m+\delta}(z), \\
& G_{0}(z), G_{1}(z), \cdots, G_{m+\delta}(z),
\end{aligned}
$$

of which the members are defined successively by the formulas

$$
\begin{aligned}
\mathcal{E}_{n}(z) & =-\varepsilon_{0}(z) \int \frac{H_{n}(\varepsilon)}{p_{0} \varepsilon_{0}} d z, \\
G_{n}(z) & =-\varepsilon_{0}(z) \int \frac{H_{n}(G)}{p_{0} \varepsilon_{0}} d z, \quad n=0,1,2, \cdots,(m+\delta),
\end{aligned}
$$

the relations (16.8) can be put into the form

$$
U_{n}(z)=\sum_{j=0}^{n}\left[k_{n-j} G_{j}(z)+c_{n-j} \mathcal{E}_{j}(z)\right] .
$$

Since $H_{0}(U) \equiv 0$, it can be seen at once, from (16.8) and (16.3), that the relation (16.11) is valid when $n=0$. We shall show that its validity for every 
integer $n$ less than a specific one, $s$, implies its validity for $n=s$. Suppose, then, that the relation (16.11) is valid for every $n$ less than $s$. The terms in the formula

$$
H_{s}(U) \equiv U_{s-2}^{\prime \prime \prime}+\sum_{l=1}^{s}\left[p_{l} U_{s-l}^{\prime}+q_{l} U_{s-l}\right]
$$

can then be evaluated by it, wherefrom it follows that

$$
\begin{aligned}
H_{8}(U)= & \sum_{i=0}^{s-2}\left[k_{s-2-i} G_{i}^{\prime \prime \prime}+c_{s-2-i} \mathcal{E}_{i}^{\prime \prime \prime}\right] \\
& +\sum_{l=1}^{\infty} \sum_{i=0}^{s-l}\left[p_{l}\left(k_{s-l-i} G_{i}^{\prime}+c_{s-l-i} \mathcal{E}_{i}^{\prime}\right)+q_{l}\left(k_{s-l-i} G_{i}+c_{s-l-i} E_{i}\right)\right] .
\end{aligned}
$$

The replacement of the summation index $i$ by $(j-2)$ in the simple sum, and by $(j-l)$ in the double sum, gives this the form

$$
\begin{aligned}
H_{\imath}(U)= & \sum_{j=2}^{\dot{2}}\left[k_{s-j} G_{j-2}^{\prime \prime \prime}+c_{\imath-j} \mathcal{E}_{j-2}^{\prime \prime \prime}\right] \\
& +\sum_{l=1}^{s} \sum_{j=l}^{\infty}\left[k_{\imath-j}\left(p_{l} G_{j-l}^{\prime}+q_{l} G_{j-l}\right)+c_{\imath-j}\left(p_{l} \mathcal{E}_{j-l}^{\prime}+q_{l} \mathcal{E}_{j-l}\right)\right] .
\end{aligned}
$$

In the simple sum the limit on $j$ may now be lowered to 1 , since only a vanishing term is thereby added, and the order of summation in the double sum may be changed. The result is the formula

$$
\begin{aligned}
H_{\imath}(U)= & \sum_{j=1}^{\dot{ }}\left\{k_{\imath-j}\left[G_{j-2}^{\prime \prime \prime}+\sum_{l=1}^{j}\left(p_{l} G_{j-l}^{\prime}+q_{l} G_{j-l}\right)\right]\right. \\
& \left.+c_{\imath-j}\left[\varepsilon_{j-2}^{\prime \prime \prime}+\sum_{l=1}^{j}\left(p_{l} \varepsilon_{j-l}^{\prime}+q_{l} \varepsilon_{j-l}\right)\right]\right\},
\end{aligned}
$$

which is, more concisely,

$$
H_{\imath}(U)=\dot{\sum}_{j=1}^{\dot{1}}\left[k_{8-j} H_{j}(G)+c_{s-j} H_{j}(\varepsilon)\right] .
$$

The substitution of this into the relation (16.8) yields, by virtue of the definitions (16.10), the equation (16.11) with $n=s$. This is what was to be shown.

17. The determination of $r(z, \lambda)$. The irregular categories 1 and 2 . With the equations (16.7) formally fulfilled, the evaluation (16.5) assumes the form

$$
L\left(u_{3}\right)=\lambda^{2}\left\{\sum_{n=0}^{m+\delta} \frac{k_{n}}{\lambda^{n}}+\sum_{n=m+\delta+1}^{2 m^{\prime \prime}} \frac{B_{n}(U)}{\lambda^{n}}\right\} .
$$


We wish to achieve this form, and to do that while retaining for its right-hand member the highest possible degree in $\lambda$. This means that we shall seek to determine the functions $U_{n}(z)$ to be analytic, with the assignment of a nonvanishing value to the earliest element of the sequence $k_{0}, k_{1}, k_{2}, \cdots$ for which such a determination can be made. The extent to which that is possible depends upon the functions (16.9), since $U_{n}(z)$ is expressed in terms of these by the formula (16.11).

The category 1. An irregular differential equation (1.1) shall be classed as in the category 1 , with respect to the integer $m$, if the functions $G_{n}(z)$ for $n=0,1, \cdots, m$, are all analytic.

In the instance of a differential equation of this category, we shall take the integer $\delta$, in the form (16.4), to be zero, and in the formulas (16.11) we shall take $k_{0}$ to be 1 , and all the other constants to be zero. The resultant formulas (16.11) are then, more simply,

$$
U_{n}(z)=G_{n}(z), \quad n=0,1, \cdots, m .
$$

This is precisely the determination of the functions $U_{n}(z)$ that was made in $\S 7$. The deductions of Part II of this paper are therefore applicable without any modifications to differential equations of the category 1 .

THEOREM 5. A differential equation (1.1) which is of the irregular category 1 with respect to the integer $m$ admits, in any pair of associated regions of $z$ and $\lambda$ a set of solutions $w_{h, j}(z), j=0,1,2,3$, which have the forms given by the Theorems $1,2,3$, and 4 , with $\delta=0$.

There are differential equations that are in the category 1 with respect to any (every) $m$. The equation

$$
w^{\mathrm{iv}}+\lambda^{2} z w^{\prime \prime}=0,
$$

is an example of such a one. For this equation

$$
L(u) \equiv u^{\prime \prime \prime}+\lambda^{2} z u^{\prime}-\lambda^{2} u,
$$

and the determination $u_{3}(z, \lambda) \equiv-1$ is possible. Thus $G_{0}(z) \equiv-1$, and $G_{n}(z) \equiv 0$, for $n \neq 0$.

On the other hand a differential equation may be in the category 1 for integers $m$ that are below a certain bound, but not for larger $m$. If the set $G_{n}(z)$ for $n=0,1,2, \cdots, m$, contains a nonanalytic member the differential equation is not in the category 1 with respect to that $m$. For many purposes the solution forms given by the Theorem 5 for some quite small integer $m$ are adequate.

It will be observed that the category 1 includes only differential equations whose limiting forms (16.1) have no actual singularity at $z=0$. For whenever there is an actual singularity the very first element of the set $G_{n}(z)$ is nonanalytic. 
The category 2. An irregular differential equation (1.1) shall be classed as in the category 2 with respect to a given $m$ if for a pair of integers $\nu$ and $\sigma$, with

$$
\nu \leqq \sigma \leqq m,
$$

the relations

$$
\begin{aligned}
& \mathcal{E}_{n}(z) \text { analytic for } n<\nu, \\
& \mathcal{E}_{\nu}(z) \text { not analytic, } \\
& G_{n}(z) \text { analytic for } n<\sigma, \\
& G_{\sigma}(z) \text { not analytic, }
\end{aligned}
$$

maintain.

The forms of the nonanalytic components of the functions $\mathcal{E}_{\nu}(z)$ and $G_{\sigma}(z)$ are easily determined. Since $H_{\nu}(\varepsilon)$ is analytic, the integrand of the formula $(16.10)$ for $\mathcal{E}_{\boldsymbol{y}}(z)$ is a ratio of two analytic functions. Its singularity is therefore a pole. The residue at this pole is nonvanishing, since $\varepsilon_{\nu}(z)$ would otherwise be analytic. The nonuniform part of the integral is therefore a constant multiple of $\log z$, and the nonanalytic part of $\varepsilon_{\nu}(z)$ is accordingly such a multiple of $\varepsilon_{0}(z) \log z$. The same conclusion is to be drawn for the function $G_{\sigma}(z)$.

In the formula (16.4) we now again take $\delta=0$. To the constants $k_{n}$ in the relations (16.11) we assign the values $k_{0}=1, k_{n}=0$ for $n \neq 0$. Then we take, in this instance, $c_{n}=0$, for $n<(\sigma-\nu)$, and leave the remaining constants $c_{n}$ to be determined in the manner to be described below. The formulas (16.11), as they are hereby reduced, are

$$
\begin{array}{lrl}
U_{n}(z) & =G_{n}(z), & \text { for } n<(\sigma-\nu), \\
U_{n}(z) & =G_{n}(z)+\sum_{j=0}^{n-\sigma+\nu} c_{n-j} \mathcal{E}_{j}(z), & \text { for }(\sigma-\nu) \leqq n \leqq m .
\end{array}
$$

The functions $U_{0}(z), U_{1}(z), \cdots, U_{\sigma-1}(z)$, all involve only analytic terms, and so are clearly analytic. The next function of the set is $U_{\sigma}(z)$, for which the formula (17.3) is

$$
U_{\sigma}(z)=G_{\sigma}(z)+\sum_{j=0}^{\nu-1} c_{\sigma-j} \mathcal{E}_{j}(z)+c_{\sigma-\nu} \mathcal{E}_{\nu}(z) .
$$

In this the only terms that are not analytic are the first and last ones; in each of these the nonanalytic component is a constant multiple of $\varepsilon_{0}(z) \log z$. The sum of these components may be made to vanish by assigning an appropriate value to $c_{\sigma-r}$. We give the constant $c_{\sigma \rightarrow-}$ this determination, and thus obtain an analytic determination of $U_{\sigma}(z)$.

We consider now the next function $U_{\sigma+1}(z)$. Since $H_{\sigma+1}(U)$ is analytic, it is to be seen from the formula (16.8) that $U_{\sigma+1}(z)$ is analytic except possibly 
for a component which is a constant multiple of $\varepsilon_{0}(z) \log z$. However the formula

$$
U_{\sigma+1}(z)=G_{\sigma+1}(z)+\sum_{j=0}^{\nu-1} c_{\sigma+1-j} \mathcal{E}_{j}(z)+c_{\sigma-\nu+1} \mathcal{E}_{\nu}(z)+c_{\sigma-\nu} \mathcal{E}_{\nu+1}(z),
$$

shows that this component is in part contributed by the term $c_{\sigma-p+1} \varepsilon_{\nu}(z)$. It can therefore be made to vanish by assigning an appropriate value to the constant $c_{\sigma \rightarrow p+1}$. We make this assignment, and thus obtain an analytic determination of $\left(^{4}\right) U_{\sigma+1}(z)$.

The reasoning that has thus been applied to the function $U_{n}(z)$ with $n=(\sigma+1)$, is now reapplicable successively with $n=(\sigma+2), \cdots, m$. It is thus found that with suitable value assignments to the constants $c_{\sigma \rightarrow,}$, $c_{\sigma-p+1}, \cdots, c_{m-\nu}$, analytic determinations of the whole set of functions $U_{n}(z)$ are obtainable. To the remaining constants $c_{n}$, namely those for $n>(m-\nu)$, we may assign the value 0 . From these determinations the evaluation (7.6) again results. The deductions proceeding therefrom in Part II of the paper require no modifications.

THEOREM 6. A differential equation (1.1) which is of the irregular category 2 with respect to the integer $m$ admits in any pair of associated regions of $z$ and $\lambda$ $a$ set of solutions $w_{h, j}(z), j=0,1,2,3$, which have the forms given by the Theorems $1,2,3$, and 4 , with $\delta=0$.

The differential equation

$$
w^{i v}+\lambda^{2} z w^{\prime \prime}+[\lambda+z] w^{\prime}+w=0
$$

is one which is of the category 1 with respect to $m=1$, and of the category 2 with respect to any $m$ that is greater than or equal to 2 . In this instance

$$
L(u) \equiv u^{\prime \prime \prime}+\lambda^{2} z u^{\prime}+\left[-\lambda^{2}+\lambda+z\right] u .
$$

The integers $\nu$ and $\sigma$ for which the assertions (17.2) apply are found to be respectively 1 and 2 .

18. The irregular category 3. An irregular differential equation (1.1) shall be classed as in the category 3 , with respect to a given integer $m$, if the assertions (17.2) apply to it for some pair of integers $\sigma$ and $\nu$, with $\sigma<\nu \leqq m$.

In the instance of a differential equation of this category, we shall assign to the integer $\delta$ in the formula (16.4) the value

$$
\delta=\nu-\sigma .
$$

Then we shall take $k_{\delta}=1$, and $k_{n}=0$, for $n \neq \delta$ in the relations (16.11) and leave

(4) It should be observed that the functions $G_{\sigma+1}(z)$ and $\mathcal{E}_{z+1}(z)$ which are present in the formula (17.4) involve nonanalytic components that are not merely constant multiples of $\mathcal{E}_{0}(z) \log z$. The reasoning given shows, however, that these components cancel out. 
the constants $c_{n}$ to be determined. The forms to which these relations are thus specialized are

$$
\begin{array}{lr}
U_{n}(z)=\sum_{j=0}^{n} c_{n-j} \varepsilon_{j}(z), & \text { for } n<\delta, \\
U_{n}(z)=G_{n-\delta}(z)+\sum_{j=0}^{n} c_{n-j} \varepsilon_{j}(z), & \text { for } \delta \leqq n \leqq m+\delta .
\end{array}
$$

In accordance with these formulas the first $\nu$ of the functions $U_{n}(z)$ are analytic, because they include only analytic terms. The next one has the formula

$$
U_{\nu}(z)=G_{\sigma}(z)+\sum_{j=0}^{p-1} c_{\nu-j} \varepsilon_{j}(z)+c_{0} \varepsilon_{\nu}(z) .
$$

In this the terms under the summation sign are analytic. Each one of the other two terms includes a nonvanishing component which is a multiple of $\varepsilon_{0}(z) \log z$. The sum of these components can be made to vanish by assigning an appropriate value to $c_{0}$, and this value is not zero since $G_{\sigma}(z)$ is in fact nonanalytic. We make this assignment, and so obtain an analytic determination of $U_{\nu}(z)$. Inasmuch as $c_{0} \neq 0$ we observe retrospectively that the first function of the set, namely $U_{0}(z)$, is not identically zero.

The reasoning whereby it may now be shown that suitable value assignments to the constants $c_{1}, c_{2}, \cdots, c_{m-\sigma}$, result in analytic determinations of the functions $U_{v+1}(z), U_{v+c}(z), \cdots, U_{m+\delta}(z)$, is precisely that which was used in $\$ 17$ in connection with differential equations of the category 2 . To the remaining constants, namely to $c_{n}$ with $n>(m-\sigma)$, we may assign the value 0 . form

The effect of these determinations is to give the relation (17.1) the explicit

$$
L\left(u_{3}\right)=\lambda^{2}\left\{\frac{1}{\lambda^{\delta}}+\sum_{n=m+\delta+1}^{2 m^{\prime}} \frac{H_{n}(U)}{\lambda^{n}}\right\} .
$$

On the basis of this the function $r(z, \lambda)$, as defined by the formula (7.7), is again a power series in $1 / \lambda$, and the relation (10.3) maintains when $\delta$ is given the value (18.1). With $\delta$ so evaluated the entire discussion of part II of this paper is applicable to the differential equations we are presently considering.

THEOREM 7. A differential equation (1.1) which is of the irregular category 3 with respect to the integer $m$ admits, in any pair of associated regions of $z$ and $\lambda$ a set of solutions $w_{h, j}(z), j=0,1,2,3$, which have the forms given by the Theorems $1,2,3$, and 4 , with $\delta=\nu-\sigma$, where $\nu$ and $\sigma$ are the integers specified by the assertions (17.2).

The method we have thus given, has been shown to yield the solution forms of all differential equations (1.1) that are of the regular type or of an 
irregular category 1,2 , or 3 . There are, however, some equations (1.1) that escape inclusion in any one of these classes. These equations are irregular, and are ones for which the function $G_{0}(z)$ is nonanalytic, whereas all members of the set $\varepsilon_{n}(z)$ for $n=1,2, \cdots, m$, are analytic irrespective of how large $m$ is chosen. The nonanalyticity of $G_{0}(z)$ excludes these equations from the categories 1 and 2, and shows that they are all of the kind whose limiting form (16.1) has an actual singularity at $z=0$. The analyticity of the functions $\varepsilon_{1}(z), \varepsilon_{2}(z), \cdots, \varepsilon_{m}(z)$, for every $m$, precludes the existence of an integer $\nu$, and thus excludes the equations from the category 3 . In every instance of this sort, the associated equation $L(u)=0$, for $m=\infty$, is formally solvable by a power series in $1 / \lambda$ with coefficients that are analytic in $z$.

An example of such an equation is

$$
w^{\text {iv }}+\left[\lambda^{2}\left(z+z^{2}\right)+\lambda\right] w^{\prime \prime}-2 \lambda^{2} w=0 .
$$

For this the equation $L(u)=0$ is

$$
u^{\prime \prime \prime}+\left[\lambda^{2}\left(z+z^{2}\right)+\lambda\right] u^{\prime}-\lambda^{2}(1+2 z) u=0,
$$

and this has the solution

$$
u=\left(z+z^{2}\right)+\frac{1}{\lambda}
$$

When, in the case of a given differential equation (1.1) for which $G_{0}(z)$ is nonalytic, no integer $\nu$ that fulfills the specification (17.2) has been found, the alternative of the existence or nonexistence of such an integer remains unresolved. Although the applicability of our method depends upon it, we have, at this moment, no practicable method for making this resolution.

19. The hydrodynamical equation. The differential equation

$$
\frac{d^{4} \psi}{d y^{4}}-2 \alpha^{2} \frac{d^{2} \psi}{d y^{2}}+\alpha^{4} \psi-i \alpha R\left\{(\omega-c)\left(\frac{d^{2} \psi}{d y^{2}}-\alpha^{2} \psi\right)-\psi \frac{d^{2} \omega}{d y^{2}}\right\}=0,
$$

which was already given above under (1.4), is central to the theory of the laminar flow of a fluid, and is therefore familiar in the literature of hydrodynamics. The symbol $R$ that appears in it stands for a Reynold's number that is ordinarily very large. $\alpha$ and $c$ are small or moderate parameters, and $\omega$, the velocity profile, is a known function of $y$ whose second derivative is ordinarily taken to be nonvanishing. The variable $y$ is directed perpendicularly to the planes bounding the flow, and the range between these planes contains a point $y_{0}$ at which the function $[\omega(y)-c]$ has a simple zero. This point (the turning point) appears to be a source of vibrations that engender turbulence. A determination of the forms of the solutions of the equation in a region about this point is therefore of the essence, in the analysis of the stability of the flow. A derivation of the differential equation (19.1) is given 
by D. Meksyn [4]. That author $[4 ; 5]$, as well as W. Wasow $[1 ; 2 ; 3]$, make studies of the equation, and give references to other such studies.

The notational changes

$$
\begin{aligned}
z & =y-y_{0}, & \omega & =\psi, \\
\lambda^{2} & =-i \alpha R \omega^{\prime}\left(y_{0}\right), & P_{0}(z) & =\frac{\omega(y)-c}{\omega^{\prime}\left(y_{0}\right)},
\end{aligned}
$$

give the equation (19.1) the form

$$
w^{\mathrm{iv}}+\lambda^{2}\left[P_{0}(z)+\frac{1}{\lambda^{2}} P_{2}\right] w^{\prime \prime}+\lambda^{2}\left[R_{0}(z)+\frac{1}{\lambda^{2}} R_{2}\right] w=0
$$

with

$$
\begin{aligned}
R_{0}(z) & =-\alpha^{2} P_{0}(z)-P_{0}^{\prime \prime}(z), \\
P_{2} & =-2 \alpha^{2}, \quad R_{2}=\alpha^{4} .
\end{aligned}
$$

The function $\omega(y)$ being unspecified, the function $P_{0}(z)$ is likewise so. We therefore take it to be given near $z=0$ by a power series

$$
P_{0}(z)=z+a z^{2}+b z^{3}+c z^{4}+\cdots .
$$

Since $Q(z, \lambda)$, and hence $Q_{0}(z)$, is in this case vanishing, the equation is of the irregular type, with $\rho=1$. To determine the applicability of our theory we shall seek to determine the equation's category.

In the instance (19.3) the differential equation (3.3) is

$$
P_{0}(z)\left(\frac{1}{\alpha_{0}}\right)^{\prime \prime}+2 P_{0}^{\prime}(z)\left(\frac{1}{\alpha_{0}}\right)^{\prime}-\alpha^{2} P_{0}(z)\left(\frac{1}{\alpha_{0}}\right)=0 .
$$

This, by virtue of the formula (19.5), has a solution

$$
\frac{1}{\alpha_{0}}=1+\frac{1}{6} \alpha^{2} z^{2}-\frac{1}{18} a \alpha^{2} z^{3}+\left[\frac{1}{120} \alpha^{4}+\frac{1}{20} a^{2} \alpha^{2}-\frac{1}{15} b \alpha^{2}\right] z^{4}+\cdots,
$$

wherefrom it follows that

$$
\alpha_{0}=1-\frac{1}{6} \alpha^{2} z^{2}+\frac{1}{18} a \alpha^{2} z^{3}+\left[\frac{7}{360} \alpha^{4}-\frac{1}{20} a^{2} \alpha^{2}+\frac{1}{15} b \alpha^{2}\right] z^{4}+\cdots
$$

The formulas (3.6) and (3.5) accordingly give

$$
\begin{aligned}
& \gamma_{0}=-\frac{1}{3} \alpha^{2} z+\frac{4}{9} a \alpha^{2} z^{2}+\left[\frac{1}{90} \alpha^{4}-\frac{49}{90} a^{2} \alpha^{2}+\frac{7}{15} b \alpha^{2}\right] z^{3}+\cdots, \\
& q_{0}=-1-2 a z-\left[\frac{2}{3} \alpha^{2}+3 b\right] z^{2}-\left[\frac{1}{3} a \alpha^{2}+4 c\right] z^{3}+\cdots,
\end{aligned}
$$


and from the formulas (16.3) it is therefore to be found that

$$
\begin{aligned}
& \mathcal{E}_{0}(z)=z+a z^{2}+\left[\frac{1}{3} \alpha^{2}+b\right] z^{3}+\left[\frac{2}{9} a \alpha^{2}+c\right] z^{4}+\cdots \\
& G_{0}(z)=-2 a \varepsilon_{0}(z) \log z-1-a z-\left[\frac{2}{3} \alpha^{2}-3 a^{2}+3 b\right] z^{2}+\cdots .
\end{aligned}
$$

We shall suppose, in the considerations of this section, that

$$
\omega^{\prime \prime}\left(y_{0}\right) \neq 0 \text {. }
$$

Then $P_{0}^{\prime \prime}(0) \neq 0$, namely, by $(19.5), a \neq 0$. The function $G_{0}(z)$ is thus nonanalytic.

The differential equation (19.3) involves only even powers of $\lambda$. Therefore, as was observed in $\S 5$, the functions $\alpha_{n}, \gamma_{n}, p_{n}, q_{n}$ and $\varepsilon_{n}$, with odd subscripts all vanish. We may therefore turn to the evaluations of $\S 5$ for $n=2$. As it is given by the appropriate formula (5.2), we see that

$$
f_{2,2}=6 \alpha_{0}^{\prime \prime}-5 p_{0} \gamma_{0}^{\prime \prime}-\left[8 p_{0}^{\prime}+4 q_{0}\right] \gamma_{0}^{\prime}-\left[3 p_{0}^{\prime \prime}+3 q_{0}^{\prime}-R_{0}\right] \gamma_{0} .
$$

Power series segments are available for all terms in this formula. Thus we find that

$$
f_{2,2}=-\frac{2}{3} \alpha^{2}-\frac{8}{3} a \alpha^{2} z+\cdots,
$$

and hence, by (5.4), that

$$
p_{2}(z)=-\frac{8}{3} \alpha^{2}-\frac{8}{3} a \alpha^{2} z+\cdots .
$$

The formulas (5.2) likewise show that

$$
\begin{aligned}
f_{1,2}= & 2 P_{2} \alpha_{0}^{\prime}-p_{2}^{\prime} \alpha_{0}+4 \alpha_{0}^{\prime \prime \prime}+\left[p_{2}-P_{2}\right]\left[p_{0}^{\prime}+q_{0}\right] \gamma_{0}-6\left[p_{0}^{\prime}+q_{0}\right] \gamma_{0}^{\prime} \\
& -4\left[p_{0}^{\prime \prime}+2 q_{0}^{\prime}\right] \gamma_{0}^{\prime}-\left[p_{0}^{\prime \prime \prime}+3 q_{0}^{\prime \prime}\right] \gamma_{0} .
\end{aligned}
$$

From this, and the fact, to be seen from (5.8), that $q_{2}(0)=f_{1,2}(0)$, we draw that

$$
q_{2}(z)=\frac{4}{3} a \alpha^{2}+\cdots .
$$

We turn now to the formula (16.6). In accordance with that

$$
H_{2}(\varepsilon)=\left[-\frac{2}{3} \alpha^{2}+6 b\right]+\left[-\frac{4}{3} a \alpha^{2}+24 c\right] z+\cdots .
$$

On the basis of this, it is readily determined, from the formula (16.10), that the nonanalytic component of $\varepsilon_{2}(z)$ is 


$$
12[a b-2 c] \varepsilon_{0}(z) \log z .
$$

This component vanishes in the exceptional cases in which $a b=2 c$, namely in which $P_{0}^{\prime \prime}(0) P_{0}^{\prime \prime \prime}(0)=P_{0}^{\text {iv }}(0)$. By $(19.2)$ these cases may be characterized otherwise as those in which $\omega^{\prime \prime}\left(y_{0}\right) \omega^{\prime \prime \prime}\left(y_{0}\right)=\omega^{\prime}\left(y_{0}\right) \omega^{\text {iv }}\left(y_{0}\right)$. In all cases, therefore, in which

$$
\omega^{\prime \prime}\left(y_{0}\right) \omega^{\prime \prime \prime}\left(y_{0}\right) \neq \omega^{\prime}\left(y_{0}\right) \omega^{\mathrm{iv}}\left(y_{0}\right)
$$

the function $\varepsilon_{2}(z)$ is nonanalytic. Any differential equation (19.1) which fulfills the conditions (19.6) and (19.9) is thus in the category 3, with $\sigma=0$ and $\nu=2$. The Theorem 7 applies to it, with $\delta=2$, and any $m$ greater than 1 .

We shall not pursue further the classification of this differential equation, with the general coefficient (19.5), in the exceptional cases in which the condition (19.9) is not fulfilled. To do that we should have to consider the analyticity of $\varepsilon_{n}(z)$ for higher subscripts $n$. That would require greater precision than is afforded by the representation of $P_{0}(z)$ through the short series segment (19.5). In the following section we shall consider a special case of the equation (19.1) in which neither of the conditions (19.6) and (19.9) is fulfilled.

20. The differential equation for Couette flow. The plane Couette flow of an incompressible viscous fluid is described by the differential equation (19.1), with $\omega$ a linear function of $y$, namely $[\omega(y)-c]=\omega^{\prime}\left(y_{0}\right)\left[y-y_{0}\right]$. By the notational changes

$$
z=\alpha\left[y-y_{0}\left[, \quad w=\psi, \quad \lambda^{2}=\frac{i R \omega^{\prime}\left(y_{0}\right)}{\alpha^{2}}\right.\right.
$$

the equation is in this instance given the completely specific form

$$
\omega^{\text {iv }}+\left[\lambda^{2} z-2\right] w^{\prime \prime}-\left[\lambda^{2} z-1\right] w=0 .
$$

This is the equation (19.3), with

$$
P_{0}(z)=z, \quad P_{2}=-2, \quad R_{0}(z)=-z, \quad R_{2}=1 .
$$

We shall show that this equation is in the category 1 , at least with respect to every integer $m$ that is less than 6 .

In the instance of the equation (20.1), the differential equation (3.3) has the analytic solution $1 / \alpha_{0}=\sinh z / z$. From this, and the formulas (3.5) and (3.6), we draw, therefore, that

$$
\begin{aligned}
& \alpha_{0}=z \operatorname{csch} z \\
& \gamma_{0}=\operatorname{csch} z-\frac{\sinh z}{z}, \\
& q_{0}=1-2 z \operatorname{coth} z .
\end{aligned}
$$

Inasmuch as the limiting form of the equation (20.1) as $\lambda \rightarrow \infty$ has no singularity, the functions $G_{0}(z)$ and $\varepsilon_{0}(z)$ are both analytic. Their formulas are 


$$
\begin{aligned}
\varepsilon_{0}(z) & =\frac{\sinh ^{2} z}{z}, \\
G_{0}(z) & =-\frac{\sinh (2 z)}{2 z} .
\end{aligned}
$$

For the calculations that are to be made, the power series representations are advantageous. In the place of the closed formulas above we shall therefore refer to their equivalents.

$$
\begin{aligned}
& \alpha_{0}=1-\frac{1}{2 \cdot 3} z^{2}+\frac{7}{2^{3} \cdot 3^{2} \cdot 5} z^{4}-\frac{31}{2^{4} \cdot 3^{3} \cdot 5 \cdot 7} z^{6}+\frac{127}{2^{7} \cdot 3^{3} \cdot 5^{2} \cdot 7} z^{8}+\cdots, \\
& \frac{1}{\alpha_{0}}=1+\frac{1}{2 \cdot 3} z^{2}+\frac{1}{2^{3} \cdot 3 \cdot 5} z^{4}+\frac{1}{2^{4} \cdot 3^{2} \cdot 5 \cdot 7} z^{6}+\frac{1}{2^{7} \cdot 3^{4} \cdot 5 \cdot 7} z^{8}+\cdots, \\
& \gamma_{0}=-\frac{1}{3} z+\frac{1}{2 \cdot 3^{2} \cdot 5} z^{3}-\frac{17}{2^{3} \cdot 3^{3} \cdot 5 \cdot 7} z^{5}+\frac{47}{2^{4} \cdot 3^{4} \cdot 5^{2} \cdot 7} z^{7}+\cdots, \\
& q_{0}=-1-\frac{2}{3} z^{2}+\frac{2}{3^{2} \cdot 5} z^{4}-\frac{4}{3^{3} \cdot 5 \cdot 7} z^{6}+\frac{2}{3^{3} \cdot 5^{2} \cdot 7} z^{8}+\cdots, \\
& \mathcal{E}_{c}=z+\frac{1}{3} z^{3}+\frac{2}{3^{2} \cdot 5} z^{5}+\frac{1}{3^{2} \cdot 5 \cdot 7} z^{7}+\cdots, \\
& \mathcal{S}_{0}=-1-\frac{2}{3} z^{2}-\frac{2}{3 \cdot 5} z^{4}-\frac{4}{3^{2} \cdot 5 \cdot 7} z^{6}+\cdots .
\end{aligned}
$$

The differential equation (20.1) involves only even powers of $\lambda$. Therefore the determinations of $\$ \S 4$ and 5 with odd subscripts all yield vanishing results, and we need consider only those for which the subscripts are even. The expression for $f_{2,2}$ has been given in (19.7). From that, it may be found that

$$
f_{2,2}=-\frac{2}{3}-\frac{43}{3^{2} \cdot 5} z^{2}+\frac{983}{2^{2} \cdot 3^{3} \cdot 5 \cdot 7} z^{4}+\cdots
$$

The relation (5.4) accordingly gives

$$
p_{2}=-\frac{8}{3}-\frac{38}{3^{2} \cdot 5} z^{2}+\frac{128}{3^{2} \cdot 5 \cdot 7} z^{4}+\cdots .
$$

With $p_{2}(z)$ thus determined, all functions entering into the formulas for $f_{0,2}$ and $f_{1,2}$ are known. The latter of these formulas was given in (19.8), and the former, as it may be drawn from (5.2) is

$$
\begin{aligned}
f_{0,2}= & P_{2} \alpha_{0}^{\prime \prime}+R_{2} \alpha_{0}+\alpha_{0}^{\text {iv }}+\left[p_{2}-P_{2}\right] q_{0}^{\prime} \gamma_{0} \\
& -6 q_{0}^{\prime} \gamma_{0}^{\prime \prime}-4 q_{0}^{\prime \prime} \gamma_{0}^{\prime}-q_{0}^{\prime \prime \prime} \gamma_{0} .
\end{aligned}
$$


In powers of $z$, these are found to proceed thus

$$
\begin{aligned}
& f_{0,2}=\frac{16}{3^{2} \cdot 5}+\frac{104}{3^{3} \cdot 5 \cdot 7} z^{2}-\frac{10534}{3^{4} \cdot 5^{2} \cdot 7} z^{4}+\cdots, \\
& f_{1,2}=-\frac{176}{3 \cdot 5 \cdot 7} z^{3}+\cdots .
\end{aligned}
$$

The calculation next before us, is that of the solution of the differential equation (5.7). For the coefficients of this equation we determine the representations

$$
\begin{aligned}
{\left[2 P_{0}^{\prime}-3 P_{0} \frac{\alpha_{0}^{\prime}}{\alpha_{0}}\right] } & =2+z^{2}+\cdots \\
{\left[f_{0,2}-f_{1,2}^{\prime}+f_{1,2} \frac{\alpha_{0}^{\prime}}{\alpha_{0}}\right] } & =\frac{16}{3^{2} \cdot 5}+\frac{4856}{3^{3} \cdot 5 \cdot 7} z^{2}+\cdots,
\end{aligned}
$$

From these the solution is found to be

$$
I_{2}=\frac{8}{3^{2} \cdot 5}+\frac{1172}{3^{3} \cdot 5 \cdot 7} z^{2}+\cdots
$$

and in terms of this latter the formulas (5.5) and (5.8) yield

$$
\begin{aligned}
& \alpha_{2}=\frac{8}{3^{2} \cdot 5} z+\frac{124}{3^{2} \cdot 5 \cdot 7} z^{8}+\cdots, \\
& q_{2}=\frac{16}{3^{2} \cdot 5} z+\frac{272}{3^{2} \cdot 5 \cdot 7} z^{3}+\cdots
\end{aligned}
$$

We may now evaluate $f_{3,2}$. This is explicitly

$$
f_{3,2}=4 \alpha_{2}^{\prime}+4 \gamma_{0}^{\prime \prime \prime}+\left[2 P_{2}-4 p_{2}\right] \gamma_{0}^{\prime}-\left[3 p_{2}^{\prime}+q_{2}\right] \gamma_{0},
$$

in accordance with which

$$
f_{3,2}=-\frac{56}{3^{2} \cdot 5}+\frac{1616}{3^{3} \cdot 5 \cdot 7} z^{2}+\cdots .
$$

It is thus to be found from (5.9) that

$$
\gamma_{2}=-\frac{28}{3^{2} \cdot 5}+\frac{68}{3^{2} \cdot 5 \cdot 7} z^{2}+\cdots .
$$

The relations to be used next are those of $\$ 16$. Recalling that all functions $p_{j}(z)$ and $q_{j}(z)$ with odd subscripts are zero, we find from (16.6) that 


$$
\begin{aligned}
& H_{2}(\mathcal{E})=-\frac{2}{3}-\frac{22}{3^{2} \cdot 5} z^{2}+\frac{584}{3^{3} \cdot 5 \cdot 7} z^{4}+\cdots, \\
& H_{2}(G)=-\frac{8}{3 \cdot 5 \cdot 7} z^{3}+\cdots .
\end{aligned}
$$

The formulas (16.10) therewith yield the representations

$$
\begin{aligned}
& \mathcal{E}_{2}=-\frac{2}{3}+\frac{2}{3^{2} \cdot 5} z^{2}-\frac{176}{3^{3} \cdot 5 \cdot 7} z^{4}+\cdots, \\
& G_{2}=\frac{4}{3 \cdot 5 \cdot 7} z^{3}+\cdots,
\end{aligned}
$$

and from these we see that the functions $\varepsilon_{2}(z)$ and $G_{2}(z)$ are both analytic.

The calculation proceeds now to the determinations of $\S 5$ for $n=4$. The formulas (5.2) that are immediately relevant are

$$
\begin{aligned}
f_{2,4}= & 6 \alpha_{2}^{\prime \prime}+\gamma_{0}^{\mathrm{i \gamma}}-\left[P_{2}-p_{2}\right]\left[p_{0} \gamma_{2}+p_{2} \gamma_{0}\right]-\left[6 p_{0}-P_{0}\right] \gamma_{2}^{\prime \prime} \\
& -\left[8 p_{0}^{\prime}+4 q_{0}\right] \gamma_{2}^{\prime}-\left[3 p_{0}^{\prime \prime}+3 q_{0}^{\prime}-R_{0}\right] \gamma_{2}+\left[P_{2}-p_{2}\right] \alpha_{2} \\
& -\left[6 p_{2}-P_{2}\right] y_{0}^{\prime \prime}-\left[8 p_{2}^{\prime}+4 q_{2}\right] \gamma_{0}^{\prime}-\left[3 p_{2}^{\prime \prime}+3 q_{2}^{\prime}-R_{2}\right] \gamma_{0},
\end{aligned}
$$

and

$$
\begin{aligned}
f_{1,4}= & -p_{4}^{\prime} \alpha_{0}+4 \alpha_{2}^{\prime \prime \prime}+\left[p_{2}-P_{2}\right]\left\{\left[p_{0}^{\prime}+q_{0}\right] \gamma_{2}+\left[p_{2}^{\prime}+q_{2}\right] \gamma_{0}\right\} \\
& +4\left[p_{0}^{\prime}+q_{0}\right] \gamma_{0}-6\left[p_{0}^{\prime}+q_{0}\right] \gamma_{2}^{\prime \prime}-4\left[p_{0}^{\prime \prime}+2 q_{0}^{\prime}\right] \gamma_{2}^{\prime} \\
& -\left[p_{0}^{\prime \prime \prime}+3 q_{0}^{\prime \prime}\right] \gamma_{2}+2 P_{2} \alpha_{2}^{\prime}-\left[p_{2}^{\prime}+q_{2}\right] \alpha_{2}-6\left[p_{2}^{\prime}+q_{2}\right] \gamma_{0}^{\prime \prime} \\
& -4\left[p_{2}^{\prime \prime}+2 q_{2}^{\prime}\right] \gamma_{0}^{\prime}-\left[p_{2}^{\prime \prime \prime}+3 q_{2}^{\prime \prime}\right] \gamma_{0} .
\end{aligned}
$$

From the expression of $f_{2,4}$ in powers of $z$ we draw, through (5.4), the evaluation

$$
p_{4}=\frac{1048}{3^{2} \cdot 5 \cdot 7} z+\cdots
$$

Thereupon $f_{1,4}$ can be evaluated, and from that the formula (5.8) leads to

$$
q_{4}=\frac{1528}{3^{3} \cdot 5 \cdot 7}+0 z+\cdots .
$$

We shall not carry the calculations much further. Indeed, since the explicitness of the series representations we have set down is almost exhausted at this point, the use of longer series segments would be requisite to carrying the calculations on. From the representations that have been derived, it is found that the power series for $H_{4}(\varepsilon)$ and $H_{4}(G)$ both lack the term in the first power of $z$. The quotients of these series by $p_{0}(z) \varepsilon_{0}(z)$ therefore have no 
terms in $1 / z$, and thus the integrals in the relations (16.10) introduce no logarithmic terms. The functions $\mathcal{E}_{4}(z)$ and $G_{4}(z)$ are thus both analytic. Since the functions $\mathcal{E}_{5}(z)$ and $G_{5}(z)$ vanish, and are therefore analytic, the differential equation (20.1) is seen to comply with the requirements for inclusion in the category 1, at least for all $m$ that are less than 6 .

\section{REFERENCES}

1. Wolfgang Wasow, $A$ study of the solutions of the differential equation $y^{(4)}+\lambda^{2}\left(x y^{\prime \prime}+y\right)=0$ for large values of $\lambda$, Ann. of Math. vol. 52 (1950) pp. 350-361.

2. - Asymptotic solution of the differential equation of hydrodynamic stability in a domain containing a transition point, Ann. of Math. vol. 58 (1953) pp. 222-252.

3. - On small disturbances of plane Couette flow, Journal of Research of the National Bureau of Standards (Washington, D. C.) vol. 51 (1953) pp. 195-202.

4. D. Meksyn, Fluid motion between parallel planes. Dynamical stability, Proc. Roy. Soc. London Ser. A vol. 186 (1946) pp. 391-409.

5. - - Asymptotic integrals of a fourth order differential equation containing a large parameter, Proc. London Math. Soc. Ser. 2 vol. 49 (1947) pp. 436-457.

6. R. E. Langer, The asymptotic solutions of ordinary linear differential equations of the second order, with special reference to a turning point, Trans. Amer. Math. Soc. vol. 67 (1949) pp. 461-490.

7. - On the asymptotic forms of the solutions of ordinary linear differential equations of the third order in a region containing a turning point, Trans. Amer. Math. Soc. vol. 80 (1955) pp. 93-123.

8. - The solutions of the differential equation $v^{\prime \prime \prime}+\lambda^{2} z v^{\prime}+3 \mu \lambda^{2} v=0$, Duke Math. J. vol. 22 (1955) pp. 525-542.

9. - The solutions of a class of ordinary linear differential equations of the third order in a region containing a multiple turning point, Duke Math. J. vol. 23 (1956) pp. 93-110.

10. - On the construction of related differential equations, Trans. Amer. Math. Soc. vol. 81 (1956) pp. 394-410.

UNIVERSITY OF WISCONSIN, Madison, Wis. 